\title{
Eruptive activity of Planchón-Peteroa volcano for period 2010-2011, Southern Andean Volcanic Zone, Chile
}

\author{
*Felipe Aguilera ${ }^{1,2}$, Óscar Benavente ${ }^{3}$, Francisco Gutiérrez ${ }^{3}$, Jorge Romero ${ }^{4}$, Ornella Saltori ${ }^{5}$, \\ Rodrigo González ${ }^{6}$, Mariano Agusto ${ }^{7}$, Alberto Caselli ${ }^{8}$, Marcela Pizarro ${ }^{5}$
}

\author{
${ }^{1}$ Servicio Nacional de Geología y Minería, Avda. Santa María 0104, Santiago, Chile. \\ ${ }^{2}$ Present address: Departamento de Ciencias Geológicas, Universidad Católica del Norte, Avda. Angamos 0610, Antofagasta, Chile. \\ feaguilera@ucn.cl \\ ${ }_{3}^{3}$ Departamento de Geología, Universidad de Chile, Plaza Ercilla 803, Santiago, Chile. \\ obenaven@ing.uchile.cl; francisco.gutierrez.ferrer@gmail.com \\ ${ }^{4}$ Centro de Investigación y Difusión de Volcanes de Chile, Proyecto Archivo Nacional de Volcanes, Santiago, Chile. \\ jorge.romerom@alumnos.uda.cl \\ ${ }^{5}$ Programa de Doctorado en Ciencias mención Geología, Universidad de Chile, Plaza Ercilla 803, Santiago, Chile. \\ o.saltori@gmail.com; marcela.pizarro@ing.uchile.cl \\ ${ }^{6}$ Departamento de Ciencias Geológicas, Universidad Católica del Norte, Avda. Angamos 0610, Antofagasta, Chile. \\ r_gonzalez@ucn.cl \\ ${ }^{7}$ Departamento de Ciencias Geológicas, Universidad de Buenos Aires, Ciudad Universitaria, Pabellón 2, 1428EHA, Buenos Aires, \\ Argentina. \\ magusto@gl.fcen.uba.ar \\ ${ }^{8}$ Laboratorio de Estudio y Seguimiento de Volcanes Activos (LESVA), Universidad Nacional de Río Negro, Roca 1242, (8332) \\ Roca, Argentina. \\ atcaselli@unrn.edu.ar
}

*Corresponding author: feaguilera@ucn.cl

\begin{abstract}
Planchón-Peteroa volcano started a renewed eruptive period between January 2010 and July 2011. This eruptive period was characterized by the occurrence of 4 explosive eruptive phases, dominated by low-intensity phreatic activity, which produced almost permanent gas/steam columns (200-800 $\mathrm{m}$ height over the active crater). Those columns presented frequently scarce ash, and were interrupted by phreatic explosions that produced ash columns 1,000-3,000 $\mathrm{m}$ height in the more intense periods. Eruptive plumes were transported in several directions (NW, N, NE, E and SE), but more than half of the time the plume axis was $130-150^{\circ} \mathrm{E}$, and reached a distance up to $638 \mathrm{~km}$ from the active crater. Tephra fall deposits identified in the NW, N, NE, E and SE flanks covered an area of $1,265 \mathrm{~km}^{2}$, thickness variable from $4 \mathrm{~m}$ (SE border of active crater) to $\sim 0.5 \mathrm{~cm} 36.8 \mathrm{~km} \mathrm{SE}$ and $\sim 8 \mathrm{~km} \mathrm{NW}$ from active crater, respectively, corresponding to a minimum volume of $0.0088 \mathrm{~km}^{3}$. Tephra fall deposit is exclusively constituted of no juvenile fragments including: lithics fragments as main component, quartz and plagioclase crystals, some oxidized lithics, and occasional presence of $\mathrm{Fe}$ oxide, and less frequently $\mathrm{Cu}$ minerals, as single fragments. We present new field-based measurements data of the geochemistry of gas/water from fumaroles and acid crater lakes, and fall deposit analysis, that integrated with the eruptive record and GOES satellite data, suggests that the eruptive period 2010-2011 has been related to an increasing of heat and mass transfer from hydrothermal-magmatic reservoirs, which would have been favoured by the formation and/or reactivation of cracks after $8.8 \mathrm{Mw}$ Maule earthquake in February 2010. This process also allowed the ascent of fluids from a shallow hydrothermal source, dominated by reduced species as $\mathrm{H}_{2} \mathrm{~S}$ and $\mathrm{CH}_{4}$, during the entire eruptive period, and the release of more oxidizing fluids from a deep magmatic reservoir, dominated by acid species as $\mathrm{SO}_{2}$, $\mathrm{HCl}$ and $\mathrm{HF}$, increasing strongly after the end of the eruptive period, probably since October 2011. The eruptive period was scored with a magnitude of 3.36 , corresponding to a VEI 1-2.
\end{abstract}


RESUMEN. Actividad eruptiva del volcán Planchón-Peteroa durante el período 2010-2011, Zona Volcánica de los Andes del Sur, Chile. El volcán Planchón-Peteroa inicia un nuevo período eruptivo entre enero de 2010 y julio de 2011, el que se caracterizó por la ocurrencia de 4 fases eruptivas de tipo explosiva, que estuvieron dominadas por actividad freática de baja intensidad que produjo constantes columnas de vapor y gas de 200 a $800 \mathrm{~m}$ de altura, con presencia frecuente de ceniza, y la ocurrencia ocasional de explosiones freáticas, que en los períodos de actividad más intensos produjeron columnas de cenizas que alcanzaron alturas entre 1.000 yf $3.000 \mathrm{~m}$ sobre el cráter activo. Las plumas eruptivas fueron transportadas en diversas direcciones (NW, N, NE, E y SE), aunque más del $50 \%$ de estas se orientaron de $130-150^{\circ} \mathrm{E}$, lanzado a distancias de hasta $638 \mathrm{~km}$. Depósitos de caída de cenizas identificados en los flancos NW, N, NE, E y SE cubrieron un área de $1.265 \mathrm{~km}^{2}$, con espesores que varían entre $4 \mathrm{~m}$ en el borde SE del cráter activo hasta $\sim 0,5 \mathrm{~cm}$ a $36,8 \mathrm{~km}$ al SE y $\sim 8 \mathrm{~km}$ NW del él, lo que equivale a un volumen mínimo de $0,0088 \mathrm{~km}^{3}$.Estos depositos se encuentran constituidos exclusivamente de fragmentos no juveniles, tales como fragmentos líticos como componente principal (algunos parcialmente oxidados), cristales de cuarzo y plagioclasa, y ocasionalmente presencia de óxidos de Fe y minerales de $\mathrm{Cu}$, estos últimos como cristales aislados y en cantidades muy escasas. En este trabajo presentamos nuevos datos de mediciones realizadas en terreno como geoquímica de gas y agua proveniente de emisiones fumarólicas y lagos cratéricos ácidos y análisis del depósito de caída, los que integrados con información de la actividad explosiva y datos satelitales GOES, sugieren que el período eruptivo 2010-2011 es producto del incremento en la transferencia de calor y masa desde reservorios hidrotermales y magmáticos, el que habría sido favorecido por la formación y/o reactivación de fracturas luego del terremoto $8,8 \mathrm{Mw}$ ocurrido en la región del Maule en febrero de 2010. Este proceso permitió el ascenso de fluidos desde una fuente hidrotermal somera, dominada por especies reducidas tales como $\mathrm{H}_{2} \mathrm{~S}$ y $\mathrm{CH}_{4}$ durante todo el período eruptivo, con suministro de fluidos más oxidados, originados en una fuente magmática profunda dominada por especies ácidas como $\mathrm{SO}_{2}, \mathrm{HCl}$ y $\mathrm{HF}$, el cual se incrementó después del período eruptivo completo, probablemente desde octubre de 2011. Este período eruptivo presenta una magnitud de 3,36, correspondiente a un Î́ndice de Explosividad Volcánica (IEV) 1-2.

Palabras clave: Explosiones freáticas, Geoquímica de fluidos, Volcán Planchón-Peteroa.

\section{Introduction}

Diverse processes have been identified and measured during and after of volcanic unrest periods, as including volcanic edifice deformation (e.g., Ayele $e t$ al., 2007; Fournier, 2007), changes in seismic activity (e.g., Chouet et al., 1994; Bryan and Sherburn, 1999), evolution of gas and water discharges compositions (e.g., Varekamp et al., 2004; Tassi et al., 2014), changes in crater lake dynamics (e.g., Christenson, 2000), variations in eruptive products emitted (e.g., Reagan et al., 1987), among others. Those processes have been related, for example, to input of new magma batches (e.g., Fischer et al., 1997; Villemant et al., 2014), magmatic-hydrothermal system dynamics variations (e.g., Vaselli et al., 2010; Tassi et al., 2013), changes in the permeability of a sealing carapace (e.g., Fischer et al., 1996) and the occurrence of earthquakes (e.g., Walter et al., 2009). Some recent examples in the Southern Volcanic Zone (SVZ) have been the eruptive periods of Copahue volcano (1992, 1995, 2000 and 2012-2014), where the deformation detected in its NE flank, changes in water composition from its crater lake and hot springs, and variations in the composition of gas released were interpreted as a consequence of intrusion of fresh magma into the hydrothermal system (Varekamp et al., 2001; Vélez et al., 2011; Agusto et al., 2013). In the case of Planchón-Peteroa volcano, the 1991 eruption was related to injection of new magma, which produced drastic morphological changes, creating two new craters (Naranjo et al., 1999), while during the last eruptive period (2010-2011), Naranjo (2012) suggested that the new eruptive cycle has a probable magmatic origin, being its eruptive products similar than the lava and pyroclasts emitted during 1837 eruption.

Planchón-Peteroa volcano $\left(35.240^{\circ} \mathrm{S}, 70.570^{\circ} \mathrm{W}\right.$, 3,603 $\mathrm{m}$ a.s.1.), located in the Transitional Southern Volcanic Zone (TSVZ; López-Escobar et al., 1995; Sellés et al., 2004), is an active composite stratovolcano situated in the centre of a NS trend volcanic chain formed by Planchón, Peteroa and Azufre volcanoes (Fig. 1; Tormey et al., 1995; Naranjo et al., 1999). A $\sim 5 \mathrm{~km}$ diameter caldera-type crater constitutes Peteroa volcano, where four nested craters and a scoria cone are hosted (Fig. 2). These craters show diameters ranging between 150 and $500 \mathrm{~m}$, and depth from 50 to $150 \mathrm{~m}$ (Naranjo and Haller, 2002). Three craters exhibit acid lakes (crater 1, 3 and 4; Fig. 2), whereas the fourth (crater 2; Fig. 2) hosts a sporadic lake whose presence 


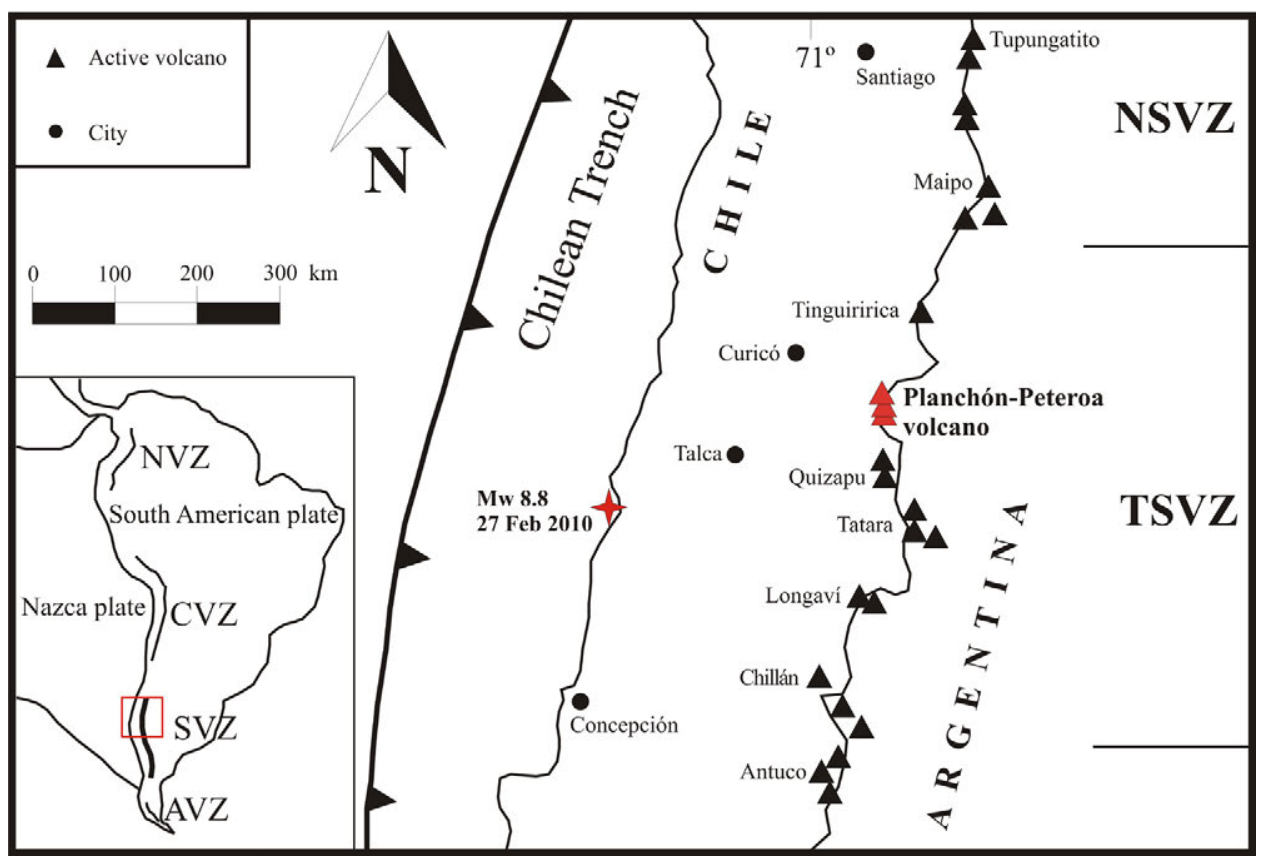

FIG. 1. Schematic map of the Southern Volcanic Zone (SVZ) and location of Planchón-Peteroa volcano. Limits of North Southern Volcanic Zone (NSVZ) and Transitional Southern Volcanic Zone (TSVZ) are shown. Localization of epicentre of $27^{\text {th }}$ February 2010 earthquake is also shown. Additionally, the distribution of others Andean volcanic zones (Northern Volcanic Zone-NVZ, Central Volcanic Zone-CVZ, Austral Volcanic Zone-AVZ) and localization of epicentre of $27^{\text {th }}$ February 2010 earthquake are also shown.

depends mainly on the rain rate and/or melt rates of the glacier located in the summit caldera (Trombotto Liaudat et al., 2014). Fumarolic activity takes place in all craters, being the fumarolic activity in crater 4 very diffuse. Additionally, a fumarolic field is present in the platform between the craters 1 and 2 (Fig. 2).

Several glaciers are hosted in the summit caldera, but also are filling the highest parts of valleys, especially in the eastern, southern and western flanks of the complex (Naranjo et al., 1999). Glaciers are highly relevant for volcanic hazards, considering that they are the primary source for lahars. The historical records show that lahars have been frequently associated with Planchón-Peteroa eruptions, having occurred in at least 4 eruptions between 1660 and 1998 (González-Ferrán, 1995; Naranjo et al., 1999; Haller and Risso, 2011). The greatest lahar in historical times occurred in March 1762, when the waters of Teno River, which run near to Teno, Romeral and Curicó towns, were stagnant during ten days as a consequence of the lahar (González-Ferrán, 1995).

This work describes the main characteristics of the recent eruptive activity of Planchón-Peteroa volcano (between January 2010 and July 2011) based on: i) field measurements (gas and water geochemistry from fumarolic emissions and crater lake waters), and ii) analysis of the fall deposit, integrated with: i) satellite data (GOES satellite imagery), ii) surveillance camera data from ICES (International Center of Earth Sciences-Argentina) and iii) local witnesses information. The aim of this work is to determine the type and magnitude of the eruption and to establish the origin and deep process related to this unrest period.

\section{Geological and volcanological setting}

The Planchón-Peteroa volcano overlies a basement constituted by Cretaceous marine and evaporitic sediments, which is composed mostly of limestone, conglomerates, sandstones and siltstones, basaltic to rhyolitic lava flows and pyroclastic rocks (Tertiary) and Middle Tertiary granodiorite plutons (Haller et al., 1985; Tormey, 1989; Tormey et al., 1995; Naranjo et al., 1999).

Tormey (1989), Tormey et al. (1995), Naranjo et al. (1999), Naranjo and Haller (2002) and Tormey (2010) have described widely the evolution of PlanchónPeteroa volcano, which is summarized as follow: 


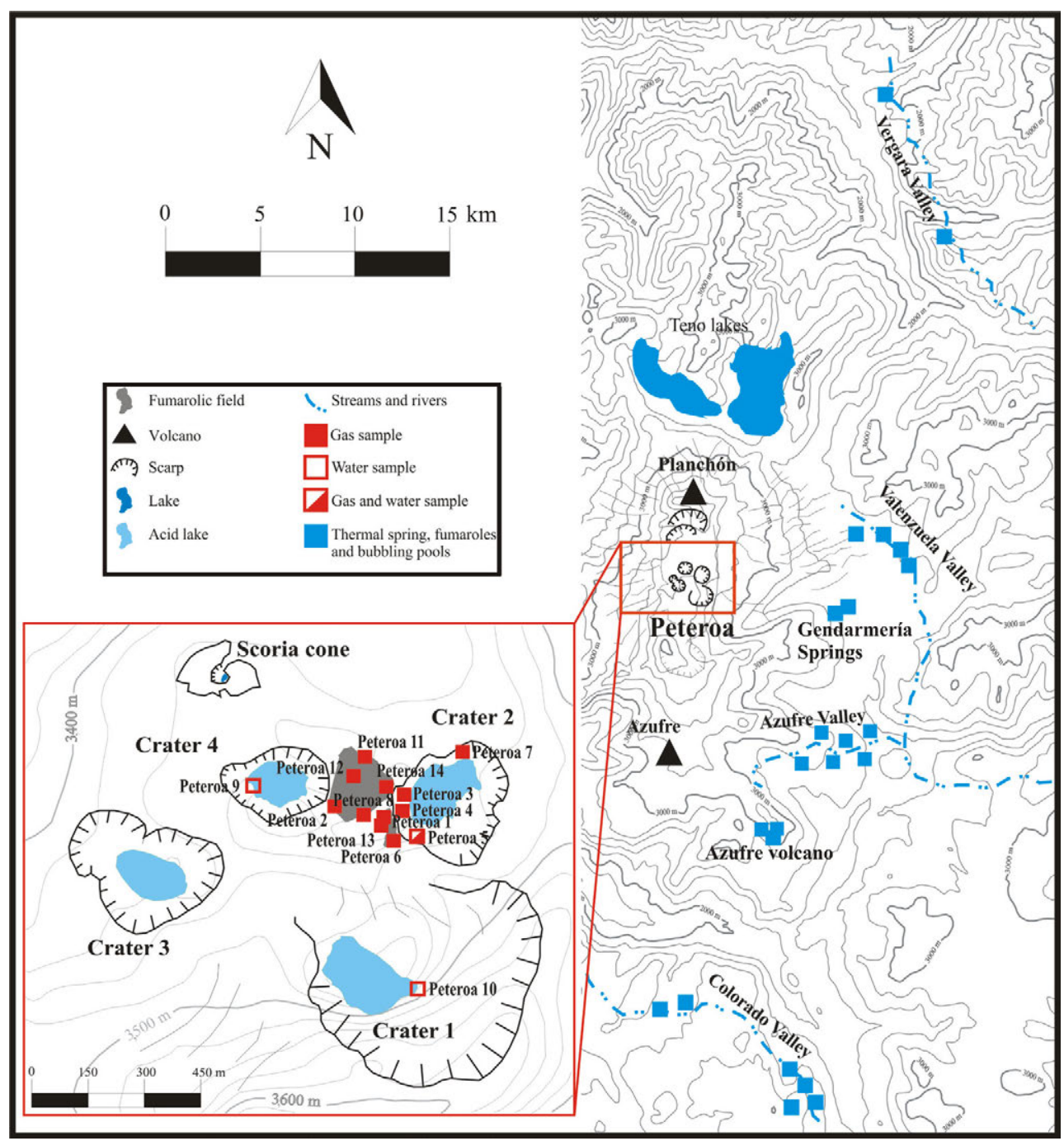

FIG. 2. Schematic map of the Planchón-Peteroa volcano, main fumarolic fields, crater lakes and location of the sampling sites are shown.

1. Azufre volcano is the earliest $(<550 \mathrm{ka})$ and largest $\left(\sim 69 \mathrm{~km}^{3}\right)$ edifice which is constituted mainly by basaltic andesite lavas and pyroclastic flows, restricted dacitic lava flows, rare andesitic lava and pyroclastic flows, and lahar deposits.

2. Planchón volcano ( $6 \mathrm{~km}$ to the north of Azufre) was built in different stages: i) Planchón I ( 14 to $\sim 11 \mathrm{ka}$ ), started its activity during the last stages of Azufre activity and is comprised by $\sim 4 \mathrm{~km}^{3}$ of basaltic and basaltic andesite lava flows and pyroclastic deposits; ii) 11 ka western flank of Azufre-Planchón complex collapsed forming a debris avalanche deposit which travelled $\sim 75 \mathrm{~km}$ to the west along Claro and Teno rivers (MacPhail,
1973; Davidson, 1974); iii) Planchón II ( 11 to $\sim 7 \mathrm{ka}$ ), built within the summit amphitheatre, is a $\sim 5 \mathrm{~km}^{3}$ edifice constituted by basaltic to basaltic andesite lava flows and pyroclastic deposits, and lahar deposits.

3. Peteroa, the smallest $\left(<1 \mathrm{~km}^{3}\right)$ and youngest edifice $(<7 \mathrm{ka})$, is a volcano formed by several scattered vents between Azufre and Planchón volcanoes. Peteroa volcanic products are characterized by andesitic lava flows and dacitic to rhyodacitic pyroclastic deposits.

Several thermal manifestations occur around Planchón-Peteroa volcano and are located $15 \mathrm{~km}$ northeast (Vergara valley), $6 \mathrm{~km}$ northeast (Valenzuela 
valley), $5.5 \mathrm{~km}$ east (Gendarmería springs), $8 \mathrm{~km}$ southeast (Azufre valley) and $20 \mathrm{~km}$ southeast (Colorado valley) from Peteroa caldera (Fig. 2). These fluid discharges correspond to springs, bubbling pools, mud pools, fumaroles and steaming ground (Benavente, 2010; Aguilera et al., 2011; Gutiérrez et al., 2012; Saltori, 2014). Additionally, fumaroles and thermal springs are present in a zone located $5 \mathrm{~km}$ southeast from Azufre volcano (Fig. 2) (Benavente, 2010; Saltori, 2014).

\section{Historical eruptive chronology}

The historical volcanic activity has been related exclusively to Peteroa volcano, and it is characterized by a permanent emission of gas from different fumarolic fields. At least 19 eruptions have been observed since 1660 , corresponding mainly to phreatic and phreatomagmatic eruptions with $\mathrm{VEI} \leq 2$ (Haller et al., 1994; Haller and Risso, 2011). A complete description of the historical eruptive events was made by González-Ferrán (1995) and Naranjo et al. (1999). The first eruption described corresponds to an explosive event during 1660 , which occurrence still is debatable, but a recognized explosive eruption was recorded during November-December 1751. The greatest historical eruption occurred $12^{\text {th }}$ March 1762 (VEI=4), when the explosive and effusive activity was followed by the partial collapse of the volcanic edifice (González-Ferrán, 1995; Haller y Risso, 2011). This collapse produced several debris avalanches and lahars, which affected the valley of Río Teno by stagnating of the river during ten days. Minor explosive eruptions were recorded in 1835 , 1837, 1842, 1860, 1869, 1872, 1878 and 1889-1894. During 1937, an explosive and effusive event formed the scoria cone, from where was emitted a single lava flow. This event was accompanied by generation of lahars. Explosive events also occurred in 1938, 1959, 1960, 1962 and 1967. In February 1991, a phreatomagmatic eruption produced an eruptive column of $1 \mathrm{~km}$ above the crater, which was dispersed to the east and southeast by $80 \mathrm{~km}$ (Naranjo and Haller, 1997, 2002). During this eruption, two new craters were formed (crater 3 and 4; Fig. 2), lahars were produced in the western flank of volcano, whereas Teno and Claro rivers were contaminated with acid waters from the active craters (BGVN, 1991; Haller et al., 1991). The last explosive eruption was recorded during November 1998 (BGVN, 1999).

\section{Methodology}

\subsection{Field measurements}

Six field campaigns were carried out during the eruptive period (February, September and October 2010, March and May 2011, and March 2012), in order to: i) obtain tephra fall deposits samples, ii) measure tephra fall deposit thickness, iii) observe the eruptive plume behaviour, and iv) sample gas and water from the fumarolic emissions and from the lakes hosted in the craters, respectively.

Fall deposits samples were obtained from the Peteroa caldera crater, and from the eastern flank of Planchón-Peteroa volcano during 2010 and 2011 campaigns. After digging a square $1 \mathrm{~m}$ by side, the fall deposit samples were stored in plastic bags and sealed to avoid the humidity generation. 500 grs. of tephra were sieved for a size range between -1 and $>3.75 \phi$. The sieved tephra was analysed with a microscope to characterize and classify the componentry, and to determine the statistical parameters associated with each tephra sample. Separation of the different fragment types was carried out using the procedure suggested by Murcia et al. (2008). From each sifter were extracted 5 grs of tephra and were deposited in glass beakers. The samples were washed with MilliQ water to clean the particles and then covered with $\mathrm{HCl} 10 \%$ vol. A magnetic bar was introduced in each beaker and shacked during $5 \mathrm{~min}$. Afterwards, each sifter was washed three times with acetone with the objective to neutralize and eliminate the $\mathrm{HCl}$, and washed again with Milli-Q water. Finally, the samples were dry in an oven at $80-100{ }^{\circ} \mathrm{C}$.

Gas sampling was performed at 8 sites within Crater 2 and fumarolic field located in the platform between craters 1 and 2 (Fig. 2). Sampling procedure was carried out in February 2010, March 2011 and March 2012. Fumarolic gases were sampled using a $1 \mathrm{~m}$ long titanium tube (diameter: $\varnothing=2.5 \mathrm{~cm}$ ) connected via a quartz-glass Dewar tubes to a pre-evacuated $60 \mathrm{~mL}$ glass thorion-tapped flasks. The flasks were filled with $20 \mathrm{~mL}$ of $4 \mathrm{~N} \mathrm{NaOH}$ and $0.15 \mathrm{M}$ $\mathrm{Cd}(\mathrm{OH})_{2}$, following the methods previously reported by Giggenbach and Goguel (1989), and Montegrossi et al. (2001). Fumarolic condensates were collected using an ice-cooled glass condensing system connected to a gas sampling glass line. Temperatures from fumarolic emissions were measured in the field after gas sampling. Crater lake water samples were 
collected from craters 1, 2 and 4 in March 2011 and March 2012 (Fig. 2). Sterile polypropylene bottles of $100 \mathrm{ml}$ were used for the cations and anions, whereas $50 \mathrm{ml}$ glass bottles were utilized for the isotopic water composition samples. Cations samples were filtered with a $0.45 \mu \mathrm{m}$ pore size membrane. No acid was added to the cations samples given the low $\mathrm{pH}$ of the water. Temperature, $\mathrm{pH}$ and conductivity were measured in the field. Gas and water samples were analysed to obtain the chemical and isotopic composition following the procedures suggested by Vaselli et al. (2006).

\subsection{Satellite images analysis}

GOES satellite images were obtained from Hawai'i Institute of Geophysics and Planetology site site (http://goes.higp.hawaii.edu last visit 3112-2013), with, with the objective to determine the direction and distance of eruptive plume. A total of 107 useful images were obtained for the period between $4^{\text {th }}$ September 2010 and $31^{\text {st }}$ May 2011, being only acquired images from 12:00 to 22:00 UTC, considering that using daylight images the eruptive plume can be better detected. Three different types of images were used (Harris et al., 2000): i) Visible, corresponds to band 1 image (visible portion of spectrum, $1 \mathrm{~km}$ pixel resolution), which is based in the measure of albedo (reflectance); ii) RGB, a false colour image where red band correspond to band 2 (mid-infrared portion of spectrum, $4 \mathrm{~km}$ pixel resolution) normalized by the mean band 2 value for the whole image, green band is the band 1 , and blue band is the band 4 inverted (thermal-infrared portion of spectrum, $4 \mathrm{~km}$ pixel resolution); iii) HOT, a false colour image similar to the RGB, differenced by green band corresponding to the algorithm defined by (band 2-band 4)/(band 2+band 4). The plume was detected in 46 visible images for 18 days, 53 RGB images for 18 days, and 8 HOT images for 4 days. Images were georeferenced using ERDAS Imagine ${ }^{\circ}$
9.1 software, with the objective to obtain an accurate measurement of the maximum distance travelled by the eruptive plumes.

\subsection{Surveillance camera images}

16 webcam images from ICES (International Center of Earth Sciences-Argentina), located $7 \mathrm{~km}$ E from Planchón-Peteroa volcano, were obtained for April, May and July 2011, which were analysed to determine eruptive plume direction and altitude.

\section{Eruptive chronology}

\subsection{Activity previous to $2010-2011$ eruptive period}

Previous to the beginning of this new eruptive period, the activity at Planchón-Peteroa was restricted to fumarolic emission in the sites previously described, and no gas column was observed (Fig. 3). During a visit in February 2009, crater lakes were present in all craters.

In the following sections are described the 4 phases defined for 2010-2011 eruptive period. The description of the phases included information of eruptive column height (meters above active crater), direction (azimuth) and distance (in $\mathrm{km}$ from active crater) reached up by the eruptive plume, places where fall of tephra was observed and dates when the fall of tephra occur. This information is summarized in the Table 1. Additionally, diverse field-based and remote-based observations are included as a complementary data.

\subsection{Phase 1}

The first eruptive phase started on $4^{\text {th }}$ January 2010, with the emission of a white column (no ash) $200 \mathrm{~m}$ over the active crater (Fig. 4a) (BGVN, 2010) which was permanent during at least 7 months (at

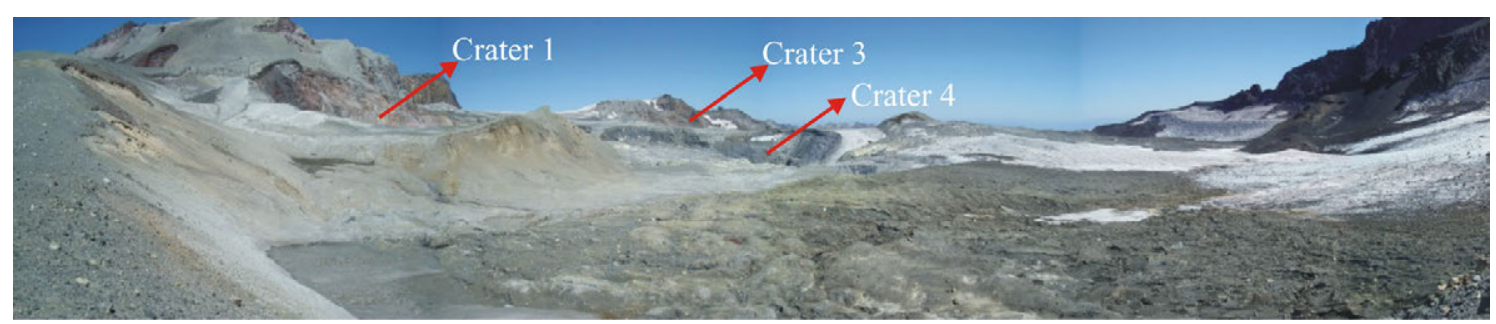

FIG. 3. Panoramic view from the east side of Peteroa caldera (February 2009). 
$7^{\text {th }}$ August 2010). During a visit between 25 and 26 February 2010, small phreatic explosions were observed in the SW border of crater 3 (Figs. 2, 4b and $\mathrm{c}$ ), which were accompanied by an intense activity in the lake (Fig. 4b and c). These phreatic explosions were partially rhythmical, with a frequency of 1 to 2 explosions every minute. The explosions produced the vaporization of the lake and probably are responsible for gas/vapour columns emission since the $4^{\text {th }}$ January 2010. During this visit, the lakes hosted in craters 1,2 and 4 did not experience any anomalous activity.

During $27^{\text {th }}$ February 2010 occurred the $\mathrm{M}_{\mathrm{w}} 8.8$ Maule earthquake, with an epicentre located $\sim 195 \mathrm{~km}$ SW from Planchón-Peteroa volcano. Despite this, no major changes in the volcano activity were observed. During $8^{\text {th }}$ August 2010, a weak light grey with scarce ash eruptive column was detected. The eruptive column reached up to $200 \mathrm{~m}$ above the active crater (crater 3 ) and was dispersed to the south. This phase was characterized by its very low activity, being classified with a $\mathrm{VEI}=0$, and a maximum $\mathrm{VEI}=1$ during the more intense period of activity (Table 1; Fig. 5).

Between the $9^{\text {th }}$ August and $3^{\text {rd }}$ September 2010 is considered a resting phase, where Planchón-Peteroa volcano activity was characterized by the presence of a very weak, but constant gas/steam white column (no ash), reaching a height of up to $100 \mathrm{~m}$ above the active crater.

\subsection{Phase 2}

On September 4 (2010) started the second eruptive phase with the emission of a grey $\sim 1,200 \mathrm{~m}$ eruptive column, which was dispersed by $30 \mathrm{~km}$ to NNE, producing fall of tephra in Río Teno Mining Company and San Pedro Custom, located 20-22 km $\mathrm{NE}$ from the active crater. Fall of tephra were also detected in different sites located 7.8 and $15 \mathrm{~km}$ $\mathrm{NE}$, and $10 \mathrm{~km} \mathrm{~N}$ from the active crater (Table 1). Between $6^{\text {th }}$ and $10^{\text {th }}$ September, grey columns, occasionally weak, and no higher than $200 \mathrm{~m}$, were dispersed to ESE at a maximum distance of $60 \mathrm{~km}$ from the active crater ( $7^{\text {th }}$ September). Fall of tephra were also seen during 6,7 and $10^{\text {th }}$ September. During the $11^{\text {th }}$ September- $11^{\text {th }}$ October 2010 period, the eruptive activity was continuous, with the emission of light to dark grey columns, whose heights varied from 200 to 3,000 m. Plumes were dispersed mainly to SE (Fig. 6a), but also to NE and NW (Fig. 6b), at the distance between 17.4 and $344.8 \mathrm{~km}$ from the active crater (Table 1). The values of VEI during these dates varied from 1 to 2, with a peak of activity during $1^{\text {st }}$ and $2^{\text {nd }}$ October (Figs. 4d and 5; Table 1). Fall of tephra occurred along SE, NE and NW flanks of Planchón-Peteroa volcano during seven days $\left(20,24,25,28\right.$ and $30^{\text {th }}$ September, $2^{\text {th }}$ and $9^{\text {th }}$ October). The area covered by the accumulated tephra deposits at $2^{\text {nd }}$ and $10^{\text {th }}$ October was 852 and $1,265 \mathrm{~km}^{2}$, respectively (Table 1). During $12^{\text {th }}$ October- ${ }^{\text {st }}$ November 2010 period the eruptive activity diminished and was characterized by the presence of a white (no ash) to light grey (very scarce ash) column, with a limited variation of height between 300 and $400 \mathrm{~m}$, except during $1^{\text {st }}$ November, when a white gas/steam plume ascent up to $1,000 \mathrm{~m}$ above active crater. During this phase, the VEI varied between 0 and 1 , whereas eruptions with VEI $=2$ where recorded in $4^{\text {th }}$ and $9^{\text {th }}$ September, and $1^{\text {st }}, 2^{\text {nd }}, 9^{\text {th }}, 10^{\text {th }}$ and $11^{\text {th }}$ October 2010 (Table 1; Fig. 5).

Anew resting phase occurred between $2^{\text {nd }}$ November 2010 and $16^{\text {th }}$ February 2011, and was characterized by the presence of a permanent white gas/steam column of a height of $200-300 \mathrm{~m} \mathrm{(2^{ \text {nd } }}$ November $2010-3^{\text {rd }}$ January 2011) and $300-650 \mathrm{~m}\left(6^{\text {th }}\right.$ January- $16^{\text {th }} \mathrm{Fe}-$ bruary 2010). During the 4 and $5^{\text {th }}$ January 2011, the columns reached up to $1,500 \mathrm{~m}$ height. No crater lake was observed in the crater 3 during $11^{\text {th }}$ November 2010 (D. Tormey, personal communication, 2011; Figs. $4 \mathrm{e}$ and 5), likely caused by a strong vaporization during eruptive phase 2 .

\subsection{Phase 3}

The $3^{\text {rd }}$ eruptive phase covers the period from the $17^{\text {th }}$ February to $20^{\text {th }}$ May 2011, and was characterized by the presence of a permanent white to grey (scarce ash) column, with heights varying between 200 and $800 \mathrm{~m}$ above the active crater. The maximum distance of eruptive plumes were observed on $26^{\text {th }}$ March, reaching up to $37 \mathrm{~km} \mathrm{SE}$ from the active crater, and during $3^{\text {rd }}$ May, when the plumes were displaced mainly to ESE, reaching a maximum travelling distance of $\sim 56.8 \mathrm{~km}$ (Table 1). Peaks of activity were recorded during a relative long time lapse $\left(17^{\text {th }}\right.$ February, $18^{\text {th }}$ March and $4^{\text {th }}$ April 2011), when grey columns, reaching heights up to $1,000-1,100 \mathrm{~m}$, being transported to the $\mathrm{SE}$ 
TABLE 1. SUMMARY OF THE ERUPTIVE CHRONOLOGY OF PLANCHÓN-PETEROA VOLCANO FOR ERUPTIVE PERIOD $2010-2011$.

\begin{tabular}{|c|c|c|c|c|c|c|c|c|c|}
\hline Date & $\begin{array}{l}\text { Eruptive } \\
\text { column } \\
\text { height }\end{array}$ & $\begin{array}{l}\text { GOES } \\
\text { Image } \\
\text { acquisi- } \\
\text { tion time }\end{array}$ & $\begin{array}{c}\text { Plume } \\
\text { dispersion }^{2}\end{array}$ & $\begin{array}{c}\text { Plume } \\
\text { distance }^{3}\end{array}$ & $\begin{array}{l}\text { Tephra } \\
\text { fall }\end{array}$ & $\begin{array}{l}\text { Tephra fall } \\
\text { sites }\end{array}$ & $\mathrm{VEI}^{4}$ & Observations & $\begin{array}{c}\text { Eruptive } \\
\text { phase }\end{array}$ \\
\hline 04-01-2010 & $200 \mathrm{~m}$ & - & - & - & - & - & 1 & $\begin{array}{l}\text { Start of eruptive } \\
\text { activity. White column, } \\
\text { no ash }\end{array}$ & \\
\hline $\begin{array}{l}23-02-10 \text { to } \\
07-08-10\end{array}$ & $100-200 \mathrm{~m}$ & - & - & - & - & - & $0-1$ & $\begin{array}{l}\text { Low intensity phreatic } \\
\text { explosions in active } \\
\text { crater during February. } \\
\text { Presence of acid lake } \\
\text { was observed in the } \\
\text { active crater during } \\
25 \text { th and } 26 \text { th February. } \\
\text { White column, no } \\
\text { ash; } 8.8 \text { MW Maule } \\
\text { earthquake ( } 27 \text { th } \\
\text { February) }\end{array}$ & 1 \\
\hline 08-08-2010 & $200 \mathrm{~m}$ & - & S & - & - & - & 1 & $\begin{array}{l}\text { Weak light grey plume } \\
\text { with scarce ash }\end{array}$ & \\
\hline $\begin{array}{l}09-08-10 \text { to } \\
03-09-10\end{array}$ & $100 \mathrm{~m} *$ & - & - & - & - & - & 0 & White column, no ash & Rest \\
\hline 04-09-2010 & $1,200 \mathrm{~m}$ & - & NNE & $21-30 \mathrm{~km}$ & $\mathrm{X}$ & $\begin{array}{l}20-22 \mathrm{~km} \mathrm{NE} \\
\text { (Río Teno } \\
\text { Mining and San } \\
\text { Pedro Custom), } \\
15 \text { and } 7.8 \mathrm{~km} \\
\mathrm{NE} \text {, and } 10 \\
\mathrm{~km} \mathrm{~N}\end{array}$ & 2 & Strong grey plume & \multirow{18}{*}{2} \\
\hline 06-09-2010 & $200 \mathrm{~m}$ & - & ESE & - & $\mathrm{X}$ & ESE & 1 & $\begin{array}{l}\text { Grey plume; } 5.2 \mathrm{Ml} \\
\text { earthquake (13.4 km } \\
\text { depth, } 9 \mathrm{~km} \text { SE from } \\
\text { Peteroa volcano) }\end{array}$ & \\
\hline 07-09-2010 & $200 \mathrm{~m}$ & - & ESE & $60 \mathrm{~km}$ & $\mathrm{X}$ & ESE & 1 & Grey plume & \\
\hline 09-09-2010 & - & - & - & - & - & - & 0 & $\begin{array}{l}\text { Weak plume with } \\
\text { scarce ash }\end{array}$ & \\
\hline $10-09-2010$ & - & - & ESE & - & $\mathrm{X}$ & ESE & 0 & $\begin{array}{l}\text { Grey plume with scarce } \\
\text { ash }\end{array}$ & \\
\hline $\begin{array}{l}11 \text { to } \\
15-09-2010\end{array}$ & $200-300 \mathrm{~m}$ & - & - & - & - & - & 1 & White column, no ash & \\
\hline $\begin{array}{l}16 \text { to } \\
18-09-2010\end{array}$ & $300-400 \mathrm{~m}$ & - & SE & - & - & - & 1 & Weak plume with ash & \\
\hline \multirow[t]{5}{*}{ 19-09-2010 } & $300-400 \mathrm{~m}$ & - & SE & - & - & - & 1 & Grey plume & \\
\hline & & 1204 UTC & $134^{\circ}$ & $214,7 \mathrm{~km}$ & - & - & - & - & \\
\hline & & 1504 UTC & $137^{\circ}$ & $36,6 \mathrm{~km}$ & - & - & - & - & \\
\hline & & 1804 UTC & $143^{\circ}$ & $94 \mathrm{~km}$ & - & - & - & - & \\
\hline & & 2104 UTC & $137^{\circ}$ & $39,6 \mathrm{~km}$ & - & - & - & - & \\
\hline $20-09-2010$ & $300-400 \mathrm{~m}$ & - & SE & - & $\mathrm{X}$ & SE & 1 & $\begin{array}{l}\text { Light grey plume with } \\
\text { ash }\end{array}$ & \\
\hline \multirow[t]{5}{*}{$21-09-2010$} & $300-400 \mathrm{~m}$ & - & SE & - & - & - & 1 & Grey plume with ash & \\
\hline & & 1204 UTC & $115^{\circ}$ & $344,8 \mathrm{~km}$ & - & - & - & - & \\
\hline & & 1504 UTC & $140^{\circ}$ & $104,7 \mathrm{~km}$ & - & - & - & - & \\
\hline & & 1804 UTC & $115^{\circ}$ & $97,8 \mathrm{~km}$ & - & - & - & - & \\
\hline & & 2104 UTC & $139^{\circ}$ & $81,8 \mathrm{~km}$ & - & - & - & - & \\
\hline
\end{tabular}


table 1 continued.

\begin{tabular}{|c|c|c|c|c|c|c|c|c|c|}
\hline Date & $\begin{array}{l}\text { Eruptive } \\
\text { column } \\
\text { height }\end{array}$ & $\begin{array}{c}\text { GOES } \\
\text { Image } \\
\text { acquisi- } \\
\text { tion time }\end{array}$ & $\begin{array}{c}\text { Plume } \\
\text { dispersion }\end{array}$ & $\begin{array}{c}\text { Plume } \\
\text { distance }^{3}\end{array}$ & $\begin{array}{l}\text { Tephra } \\
\text { fall }\end{array}$ & $\begin{array}{l}\text { Tephra fall } \\
\text { sites }\end{array}$ & VEI $^{4}$ & Observations & $\begin{array}{c}\text { Eruptive } \\
\text { phase }\end{array}$ \\
\hline $\begin{array}{l}22 \text { to } \\
23-09-2010\end{array}$ & $300-400 \mathrm{~m}$ & - & SE & - & - & - & 1 & Weak plume with ash & \\
\hline \multirow[t]{3}{*}{ 24-09-2010 } & $1,000 \mathrm{~m}$ & - & SE & - & $\mathrm{X}$ & SE & 2 & Grey plume with ash & \\
\hline & - & 1204 UTC & $154^{\circ}$ & $177,6 \mathrm{~km}$ & - & - & - & - & \\
\hline & - & 1504 UTC & $177^{\circ}$ & $31,5 \mathrm{~km}$ & - & - & - & - & \\
\hline \multirow[t]{4}{*}{$25-09-2010$} & $500 \mathrm{~m}$ & - & SE & - & $\mathrm{X}$ & SE & 1 & Grey plume with ash & \\
\hline & - & 1204 UTC & $143^{\circ}$ & $204,8 \mathrm{~km}$ & - & - & - & - & \\
\hline & - & 1504 UTC & $142^{\circ}$ & $167,5 \mathrm{~km}$ & - & - & - & - & \\
\hline & - & 1804 UTC & $153^{\circ}$ & $56,6 \mathrm{~km}$ & - & - & & & \\
\hline \multirow[t]{4}{*}{ 26-09-2010 } & $300-400 \mathrm{~m}$ & - & SE & - & - & - & 1 & Grey plume & \\
\hline & - & 1204 UTC & $167^{\circ}$ & $141,7 \mathrm{~km}$ & - & - & - & - & \\
\hline & - & 1504 UTC & $167^{\circ}$ & $58,2 \mathrm{~km}$ & - & - & - & - & \\
\hline & - & 1804 UTC & $172^{\circ}$ & $43,8 \mathrm{~km}$ & - & - & - & - & \\
\hline 27-09-2010 & $300-400 \mathrm{~m}$ & - & SE & - & - & - & 1 & Weak plume with ash & \\
\hline \multirow[t]{2}{*}{ 28-09-2010 } & $300-400 \mathrm{~m}$ & - & SE, NE & - & $\mathrm{X}$ & SE, NE & 1 & Grey plume with ash & \\
\hline & - & 2104 UTC & $134^{\circ}$ & $31,4 \mathrm{~km}$ & - & - & - & - & \\
\hline 29-09-2010 & $300-400 \mathrm{~m}$ & - & SE & - & - & - & 1 & Weak plume with ash & \\
\hline 30-09-2010 & $300 \mathrm{~m}$ & - & $\mathrm{NE}$ & $\begin{array}{l}\text { tens of } \\
\mathrm{kms}\end{array}$ & $\mathrm{X}$ & NE & 1 & Light grey plume & \\
\hline \multirow[t]{4}{*}{$01-10-2010$} & $1,000 \mathrm{~m}$ & - & NW & - & - & - & 2 & $\begin{array}{l}\text { Dark grey plume with } \\
\text { abundant ash }\end{array}$ & \\
\hline & - & 1204 UTC & $340^{\circ}$ & $98,9 \mathrm{~km}$ & - & - & - & - & \\
\hline & - & 1504 UTC & $323^{\circ}$ & $64,1 \mathrm{~km}$ & - & - & - & - & 2 \\
\hline & - & 1804 UTC & $338^{\circ}$ & $254 \mathrm{~km}$ & - & - & - & - & \\
\hline \multirow[t]{5}{*}{$02-10-2015$} & $1,000-3,000 \mathrm{~m}$ & - & NNW & - & $\mathrm{X}$ & NW & 2 & $\begin{array}{l}\text { Dark grey plume with } \\
\text { ash }\end{array}$ & \\
\hline & - & 1204 UTC & $0^{\circ}$ & $19,4 \mathrm{~km}$ & - & - & - & - & \\
\hline & - & 1504 UTC & $322^{\circ}$ & $78,6 \mathrm{~km}$ & - & - & - & $\begin{array}{l}\text { Tephra fall deposit: } \\
852,23 \mathrm{~km}^{2}\end{array}$ & \\
\hline & - & 1804 UTC & $298^{\circ}$ & $72,4 \mathrm{Km}$ & - & - & - & - & \\
\hline & - & 2104 UTC & $304^{\circ}$ & $150,9 \mathrm{~km}$ & - & - & - & - & \\
\hline $\begin{array}{l}03 \text { to } \\
06-10-2010\end{array}$ & $300-400 \mathrm{~m}$ & - & SE & - & - & - & 1 & Weak plume with ash & \\
\hline \multirow[t]{4}{*}{$07-10-2010$} & $300-400 \mathrm{~m}$ & - & SE & - & - & - & 1 & Grey plume with ash & \\
\hline & - & 1504 UTC & $149^{\circ}$ & $39,7 \mathrm{~km}$ & - & - & - & - & \\
\hline & - & 1804 UTC & $154^{\circ}$ & $52,7 \mathrm{~km}$ & - & - & - & - & \\
\hline & - & 2104 UTC & $148^{\circ}$ & $48,3 \mathrm{~km}$ & - & - & - & - & \\
\hline \multirow[t]{3}{*}{ 08-10-2010 } & $300-400 \mathrm{~m}$ & - & $\mathrm{SE}$ & - & - & - & 1 & Grey plume & \\
\hline & - & 1204 UTC & $149^{\circ}$ & $26,8 \mathrm{~km}$ & - & - & - & - & \\
\hline & - & 1504 UTC & $153^{\circ}$ & $18,6 \mathrm{~km}$ & - & - & - & - & \\
\hline \multirow[t]{4}{*}{ 09-10-2010 } & $1,500 \mathrm{~m}$ & - & SE & - & $\mathrm{X}$ & $\mathrm{SE}$ & 2 & $\begin{array}{l}\text { Light grey plume with } \\
\text { ash }\end{array}$ & \\
\hline & - & 1204 UTC & $154^{\circ}$ & $19,9 \mathrm{~km}$ & - & - & - & - & \\
\hline & - & 1504 UTC & $162^{\circ}$ & $42,7 \mathrm{~km}$ & - & - & - & - & \\
\hline & - & 1804 UTC & $148^{\circ}$ & $26 \mathrm{~km}$ & - & - & - & - & \\
\hline
\end{tabular}


table 1 continued.

\begin{tabular}{|c|c|c|c|c|c|c|c|c|c|}
\hline Date & $\begin{array}{l}\text { Eruptive } \\
\text { column } \\
\text { height }\end{array}$ & $\begin{array}{l}\text { GOES } \\
\text { Image } \\
\text { acquisi- } \\
\text { tion time }\end{array}$ & $\begin{array}{c}\text { Plume } \\
\text { dispersion }^{2}\end{array}$ & $\begin{array}{c}\text { Plume } \\
\text { distance }^{3}\end{array}$ & $\begin{array}{l}\text { Tephra } \\
\text { fall }\end{array}$ & $\begin{array}{l}\text { Tephra fall } \\
\text { sites }\end{array}$ & VEI $^{4}$ & Observations & $\begin{array}{c}\text { Eruptive } \\
\text { phase }\end{array}$ \\
\hline \multirow[t]{4}{*}{$10-10-2010$} & $2,000 \mathrm{~m}$ & - & NW & - & - & - & 2 & Grey plume & \multirow{12}{*}{2} \\
\hline & & 1204 UTC & $317^{\circ}$ & $17,4 \mathrm{~km}$ & - & - & - & - & \\
\hline & & 1504 UTC & $316^{\circ}$ & $20,5 \mathrm{~km}$ & - & - & - & $\begin{array}{l}\text { Tephra fall deposit: } \\
1,265 \mathrm{~km}^{2}\end{array}$ & \\
\hline & & 1804 UTC & $321^{\circ}$ & 17,4 km & - & - & - & - & \\
\hline $11-10-2010$ & $1,000 \mathrm{~m}$ & - & NW & - & - & - & 2 & White plume with ash & \\
\hline $\begin{array}{l}12 \text { to } \\
15-10-2010\end{array}$ & $300-400 \mathrm{~m}$ & - & $\mathrm{SE}$ & - & - & - & 1 & Weak plume with ash & \\
\hline $\begin{array}{l}16 \text { to } \\
23-10-2010\end{array}$ & $300-400 \mathrm{~m} *$ & - & $\mathrm{SE}$ & - & - & - & 0 & White column, no ash & \\
\hline $24-10-2010$ & $300-400 \mathrm{~m}$ & - & $\mathrm{NE}$ & - & - & - & 1 & White column, with ash & \\
\hline $\begin{array}{l}25 \text { to } \\
29-10-2010\end{array}$ & $300-400 \mathrm{~m} *$ & - & $\mathrm{SE}$ & - & - & - & 0 & White column, no ash & \\
\hline $30-10-2010$ & $300-400 \mathrm{~m}$ & - & $\mathrm{E}$ & - & - & - & 1 & Light grey column & \\
\hline $31-10-2010$ & $300-400 \mathrm{~m}$ & - & $\mathrm{NE}$ & - & - & - & 1 & Light grey column & \\
\hline 01-11-2010 & $1,000 \mathrm{~m} *$ & - & Vertical & - & - & - & 0 & White column, no ash & \\
\hline $\begin{array}{l}02-11-2010 \\
\text { to } \\
03-01-2011\end{array}$ & $200-300 \mathrm{~m} *$ & - & $\mathrm{NE}$ & - & - & - & 0 & $\begin{array}{l}\text { White column, no ash. } \\
\text { Crater lake was not } \\
\text { observed during 11th } \\
\text { November in the active } \\
\text { crater }\end{array}$ & \multirow{3}{*}{ Rest } \\
\hline $\begin{array}{l}04 \text { to } \\
05-01-2011\end{array}$ & $1,000-1,500 \mathrm{~m} *$ & - & - & - & - & - & 0 & White column, no ash & \\
\hline $\begin{array}{l}06-01 \text { to } \\
16-02-2011\end{array}$ & $300-650 \mathrm{~m} *$ & - & - & - & - & - & 0 & White column, no ash & \\
\hline $17-02-2011$ & $1,100 \mathrm{~m}$ & - & $\mathrm{SE}$ & - & $\mathrm{X}$ & $\mathrm{SE}$ & 2 & Grey column & \multirow{13}{*}{3} \\
\hline $\begin{array}{l}18-02 \text { to } \\
17-03-2011\end{array}$ & $650-800 \mathrm{~m} *$ & - & SE & - & - & - & 0 & $\begin{array}{l}\text { White column, no ash. } \\
\text { Crater lake was obser- } \\
\text { ved during 8th March } \\
\text { in the active crater }\end{array}$ & \\
\hline $18-03-2011$ & $1,000 \mathrm{~m}$ & - & - & - & - & - & 2 & $\begin{array}{l}\text { White column, scarce } \\
\text { ash }\end{array}$ & \\
\hline $\begin{array}{l}19 \text { to } \\
25-03-2011\end{array}$ & $650 \mathrm{~m} *$ & - & - & - & - & - & 0 & White column, no ash & \\
\hline \multirow[t]{3}{*}{$26-03-2011$} & $650 \mathrm{~m}$ & - & $\mathrm{SE}$ & - & - & - & 1 & Grey column & \\
\hline & & 1504 UTC & $141^{\circ}$ & $30,8 \mathrm{~km}$ & - & - & - & - & \\
\hline & & 1804 UTC & $136^{\circ}$ & $36,9 \mathrm{~km}$ & - & - & - & - & \\
\hline $\begin{array}{l}27-03 \text { to } \\
03-04-2011\end{array}$ & $600-650 \mathrm{~m} *$ & - & $\mathrm{SE}$ & - & - & - & 0 & White column, no ash & \\
\hline 04-04-2011 & $1,000 \mathrm{~m}$ & - & $\mathrm{N}$ & - & $\mathrm{X}$ & $\mathrm{N}$ & 2 & Grey column & \\
\hline $\begin{array}{l}05 \text { to } \\
11-04-2011\end{array}$ & $600 \mathrm{~m} *$ & - & $\mathrm{SE}$ & - & - & - & 0 & White column, no ash & \\
\hline 12-04-2011 & $600 \mathrm{~m}$ & - & SE & - & - & - & 1 & Grey column & \\
\hline $\begin{array}{l}13 \text { to } \\
15-04-2011\end{array}$ & $600 \mathrm{~m} *$ & - & $\mathrm{SE}$ & - & - & - & 0 & White column, no ash & \\
\hline 16-04-2011 & $600 \mathrm{~m}$ & - & $\mathrm{SE}$ & - & - & - & 1 & $\begin{array}{l}\text { White column, scarce } \\
\text { ash }\end{array}$ & \\
\hline
\end{tabular}


table 1 continued.

\begin{tabular}{|c|c|c|c|c|c|c|c|c|c|}
\hline Date & $\begin{array}{l}\text { Eruptive } \\
\text { column } \\
\text { height }\end{array}$ & $\begin{array}{l}\text { GOES } \\
\text { Image } \\
\text { acquisi- } \\
\text { tion time }\end{array}$ & $\begin{array}{c}\text { Plume } \\
\text { dispersion }^{2}\end{array}$ & $\begin{array}{c}\text { Plume } \\
\text { distance }^{3}\end{array}$ & $\begin{array}{l}\text { Tephra } \\
\text { fall }\end{array}$ & $\begin{array}{l}\text { Tephra fall } \\
\text { sites }\end{array}$ & VEI $^{4}$ & Observations & $\begin{array}{l}\text { Eruptive } \\
\text { phase }\end{array}$ \\
\hline $\begin{array}{l}17 \text { to } \\
18-04-2011\end{array}$ & $1,200 \mathrm{~m}$ & - & SE & - & - & - & 2 & $\begin{array}{l}\text { White column, scarce } \\
\text { ash }\end{array}$ & \\
\hline 19-04-2011 & $600 \mathrm{~m}$ & - & $\mathrm{SE}$ & - & - & - & 1 & $\begin{array}{l}\text { White column, scarce } \\
\text { ash }\end{array}$ & \\
\hline 20-04-2011 & $600 \mathrm{~m} *$ & - & SE & - & - & - & 0 & White column, no ash & \\
\hline 21-04-2011 & $600 \mathrm{~m}$ & - & SE & - & - & - & 1 & $\begin{array}{l}\text { White column, scarce } \\
\text { ash }\end{array}$ & \\
\hline $\begin{array}{l}22 \text { to } \\
24-04-2011\end{array}$ & $600 \mathrm{~m} *$ & - & SE & - & - & - & 0 & White column, no ash & \\
\hline $\begin{array}{l}25 \text { to } \\
28-04-2011\end{array}$ & $600 \mathrm{~m}$ & - & SE & - & - & - & 1 & $\begin{array}{l}\text { White column, scarce } \\
\text { ash }\end{array}$ & \\
\hline 29-04-2011 & $1,200 \mathrm{~m}$ & - & SE & - & - & - & 2 & $\begin{array}{l}\text { White column, scarce } \\
\text { ash }\end{array}$ & \\
\hline 01-05-2011 & $1,500 \mathrm{~m}$ & - & SE, E, NE & - & - & - & 2 & Grey column & \\
\hline 02-05-2011 & $1,000 \mathrm{~m}$ & - & $\mathrm{NE}$ & - & $\mathrm{X}$ & NE & 2 & Grey column & \\
\hline \multirow[t]{2}{*}{ 03-05-2011 } & $500-700 \mathrm{~m}$ & - & ESE & - & $\mathrm{X}$ & ESE & 1 & Grey column & \\
\hline & & $\begin{array}{l}1204 \text { UTC } \\
1334 \text { UTC } \\
1404 \text { UTC } \\
1434 \text { UTC } \\
1504 \text { UTC }\end{array}$ & $\begin{array}{l}115^{\circ} \\
132^{\circ} \\
132^{\circ} \\
132^{\circ} \\
124^{\circ}\end{array}$ & $\begin{array}{l}12,3 \mathrm{~km} \\
31,8 \mathrm{~km} \\
46,5 \mathrm{~km} \\
56,8 \mathrm{~km} \\
40,8 \mathrm{~km}\end{array}$ & $\begin{array}{l}- \\
- \\
- \\
- \\
-\end{array}$ & $\begin{array}{l}- \\
- \\
- \\
- \\
-\end{array}$ & $\begin{array}{l}- \\
- \\
- \\
- \\
-\end{array}$ & $\begin{array}{l}- \\
- \\
- \\
- \\
-\end{array}$ & \\
\hline \multirow[t]{2}{*}{ 04-05-2011 } & $1,000 \mathrm{~m}$ & - & NW-N-NE & - & $\mathrm{X}$ & $\begin{array}{l}\text { San Pedro } \\
\text { Custom and Rio } \\
\text { Teno Mining }\end{array}$ & 2 & $\begin{array}{l}\text { Grey column, scarce } \\
\text { ash. Turbidity detection } \\
\text { along Claro river }\end{array}$ & 3 \\
\hline & & $\begin{array}{l}1504 \text { UTC } \\
1804 \text { UTC } \\
2104 \text { UTC }\end{array}$ & $\begin{array}{l}345^{\circ} \\
002^{\circ} \\
041^{\circ}\end{array}$ & $\begin{array}{l}64 \mathrm{~km} \\
41,3 \mathrm{~km} \\
17,6 \mathrm{~km}\end{array}$ & $\begin{array}{l}- \\
- \\
-\end{array}$ & $\begin{array}{l}- \\
- \\
-\end{array}$ & $\begin{array}{l}- \\
- \\
-\end{array}$ & $\begin{array}{l}- \\
- \\
-\end{array}$ & \\
\hline \multirow[t]{2}{*}{ 05-05-2011 } & $500 \mathrm{~m}$ & - & SE & - & $\mathrm{X}$ & $\begin{array}{l}8 \mathrm{~km} \mathrm{NW} \\
\text { (Carrizales), } \\
46 \mathrm{~km} \text { ENE } \\
\text { (Las Leñas), SE }\end{array}$ & 1 & $\begin{array}{l}\text { Grey column. Turbity } \\
\text { along Claro river }\end{array}$ & \\
\hline & & 1504 UTC & $145^{\circ}$ & $30 \mathrm{~km}$ & - & - & - & - & \\
\hline \multirow[t]{2}{*}{ 06-05-2011 } & $500 \mathrm{~m}$ & - & ESE & - & $\mathrm{X}$ & ESE & 1 & Grey column & \\
\hline & & $\begin{array}{l}1204 \text { UTC } \\
1504 \text { UTC } \\
1804 \text { UTC } \\
2104 \text { UTC }\end{array}$ & $\begin{array}{l}089^{\circ} \\
145^{\circ} \\
146^{\circ} \\
095^{\circ}\end{array}$ & $\begin{array}{c}373,5 \mathrm{~km} \\
114 \mathrm{~km} \\
93,1 \mathrm{~km} \\
637,6 \mathrm{~km} \\
\end{array}$ & $\begin{array}{l}- \\
- \\
- \\
-\end{array}$ & $\begin{array}{l}- \\
- \\
- \\
-\end{array}$ & $\begin{array}{l}- \\
- \\
- \\
-\end{array}$ & $\begin{array}{l}- \\
- \\
- \\
-\end{array}$ & \\
\hline 07-05-2011 & $200-300 \mathrm{~m} *$ & - & SE & - & - & - & 0 & $\begin{array}{l}\text { White column, no ash. } \\
\text { Tephra fall deposit: } \\
692,84 \mathrm{~km}^{2}\end{array}$ & \\
\hline $\begin{array}{l}08 \text { to } \\
17-05-2011\end{array}$ & $200-300 \mathrm{~m}$ & - & SE & - & $\mathrm{X}$ & $\begin{array}{l}\text { SE (only } \\
09-05-11 \text { ) }\end{array}$ & 1 & $\begin{array}{l}\text { White column, scarce } \\
\text { ash }\end{array}$ & \\
\hline $\begin{array}{l}18 \text { to } \\
19-05-2011\end{array}$ & $200-300 \mathrm{~m} *$ & - & - & - & - & - & 0 & White column, no ash & \\
\hline $20-05-2011$ & $200-300 \mathrm{~m}$ & - & $\mathrm{SE}$ & - & - & - & 1 & Light grey column & \\
\hline $\begin{array}{l}21-05 \text { to } \\
16-06-2011\end{array}$ & $200-400 \mathrm{~m} *$ & - & SE & - & - & - & 0 & White column, no ash & Rest \\
\hline
\end{tabular}


table 1 continued.

\begin{tabular}{|c|c|c|c|c|c|c|c|c|c|}
\hline Date & $\begin{array}{l}\text { Eruptive } \\
\text { column } \\
\text { height }\end{array}$ & $\begin{array}{c}\text { GOES } \\
\text { Image } \\
\text { acquisi- } \\
\text { tion time }\end{array}$ & $\begin{array}{c}\text { Plume } \\
\text { dispersion }\end{array}$ & $\begin{array}{c}\text { Plume } \\
\text { distance }^{3}\end{array}$ & $\begin{array}{l}\text { Tephra } \\
\text { fall }\end{array}$ & $\begin{array}{l}\text { Tephra fall } \\
\text { sites }\end{array}$ & $\mathrm{VEI}^{4}$ & Observations & $\begin{array}{c}\text { Eruptive } \\
\text { phase }\end{array}$ \\
\hline $17-06-2011$ & $400-500 \mathrm{~m}$ & - & - & - & - & - & 1 & $\begin{array}{l}\text { White column, scarce } \\
\text { ash }\end{array}$ & \multirow{9}{*}{4} \\
\hline $\begin{array}{l}22 \text { to } \\
26-06-2011\end{array}$ & $400-500 \mathrm{~m}$ & - & - & - & - & - & 1 & $\begin{array}{l}\text { White column, scarce } \\
\text { ash }\end{array}$ & \\
\hline 27-06-2011 & $2,000 \mathrm{~m}$ & - & $\mathrm{NE}$ & $41 \mathrm{~km}$ & - & - & 2 & Grey column & \\
\hline $30-06-2011$ & $400-500 \mathrm{~m}$ & - & - & - & - & - & 1 & $\begin{array}{l}\text { White column, scarce } \\
\text { ash }\end{array}$ & \\
\hline 01-07-2011 & $500 \mathrm{~m}$ & - & $\mathrm{NE}$ & - & - & - & 1 & Grey column & \\
\hline 04-07-2011 & $200-300 \mathrm{~m}$ & - & SE & - & - & - & 1 & $\begin{array}{l}\text { White column, scarce } \\
\text { ash }\end{array}$ & \\
\hline 05-07-2011 & $500 \mathrm{~m}$ & - & $\mathrm{SE}$ & - & - & - & 1 & Light grey column & \\
\hline $11-07-2011$ & $1,000 \mathrm{~m}$ & - & SE & - & $\mathrm{X}$ & SE & 2 & Grey column & \\
\hline 13-07-2011 & $500 \mathrm{~m}$ & - & SE & - & - & - & 1 & Light grey column & \\
\hline
\end{tabular}

Information of date, eruptive column height (meters above active crater), acquisition time for GOES satellite image, direction and distance of eruptive plumes, dates and places where fall of tephra was detected, Volcanic Explosivity Index (VEI), field observations and eruptive phases is shown. Information was collected from surveillance data from ICES (International Center of Earth SciencesArgentina), satellite data (GOES satellite imagery), information from local witnesses and field-based data. ${ }^{1}$ UTC time; ${ }^{2}$ in azimuthal convention; ${ }^{3}$ distance (in $\mathrm{km}$ from the active crater) calculated from GOES satellite images; ${ }^{4}$ VEI based exclusively in the eruptive column height (after Newhall and Self, 1982); * Presence of gas column, not eruptive column/plume.

and N. Fall of tephra occurred along the SE and $\mathrm{N}$ flanks of Planchón-Peteroa volcano during the $17^{\text {th }}$ February (Fig. 4f) and $4^{\text {th }}$ April, respectively. A new increment of the volcano activity (Table 1; Fig. 5) was reported in a short time lapse $\left(17^{\text {th }}\right.$, $18^{\text {th }}$ and $29^{\text {th }}$ April, $1^{\text {st }}, 2^{\text {nd }}, 4^{\text {th }}$ and $6^{\text {th }}$ May 2011). This activity was characterized by the emission of white to grey columns, with heights varying from 500 to $1,200 \mathrm{~m}$, and plumes transported to NW, N, NE, NE, E and SE (Fig. 6c and d), reaching up to $637.6 \mathrm{~km}$ distance from the active crater $\left(6^{\text {th }}\right.$ May; Table 1$)$. During $2^{\text {nd }}, 4^{\text {th }}$ and $6^{\text {th }}$ May fall of tephra were detected on the NW, N, NE, NE and ESE flanks of Planchón-Peteroa volcano and, Río Teno Mining Company and San Pedro Custom. Claro River, located in the W side of PlanchónPeteroa volcano, became turbid during $4^{\text {th }}$ and $5^{\text {th }}$ May. The area covered by the accumulated tephra deposit at $7^{\text {th }}$ October was $693 \mathrm{~km}^{2}$. Additionally, fall of tephra occurred during the $3^{\text {rd }}, 5^{\text {th }}$ and $9^{\text {th }}$ May 2011, in the NW, ENE and SE flanks and in Carrizales $(\sim 8 \mathrm{~km} \mathrm{NW})$ and Las Leñas $(\sim 46 \mathrm{~km}$ ENE). VEI during phase 3 varied between 0 and 1 , reaching up to 2 during $17^{\text {th }}$ February, $18^{\text {th }}$ March, $4^{\text {th }}, 17^{\text {th }}, 18^{\text {th }}$ and $29^{\text {th }}$ April, and $1^{\text {st }}, 2^{\text {nd }}$ and $4^{\text {th }}$ May 2011 (Table 1; Fig. 5).

Field observations during the $8^{\text {th }}$ March indicate the presence of a low altitude white column $(\sim 100 \mathrm{~m}$; Fig. $4 \mathrm{~g}$ ), as well as the presence of a lake in the crater 3 . However, the volume o the crater lake was lower than the one observed on February 2010 (early phase 1). A crater lake was not present in the crater 2 during the $8^{\text {th }}$ March (only presence of acid bubbling pools in the SW side), whereas crater 1 and 4 presented no changes respect to the observation carried out during the phase 1 (February 2010).

A resting phase occurred between $21^{\text {st }}$ May and $16^{\text {th }}$ June 2011, characterized by the presence of a constant very weak gas/steam white column, with a height that varied from 200 to $400 \mathrm{~m}$.

\subsection{Phase 4}

The last eruptive phase $\left(17^{\text {th }}\right.$ June-1 $13^{\text {th }}$ July 2011$)$ was characterized by permanent white to grey columns reaching up 200-500 $\mathrm{m}$ height (VEI=1). The two last explosions occurred during the $27^{\text {th }}$ June (grey column 2,000 m height displaced to NE by $41 \mathrm{~km}$ ) 


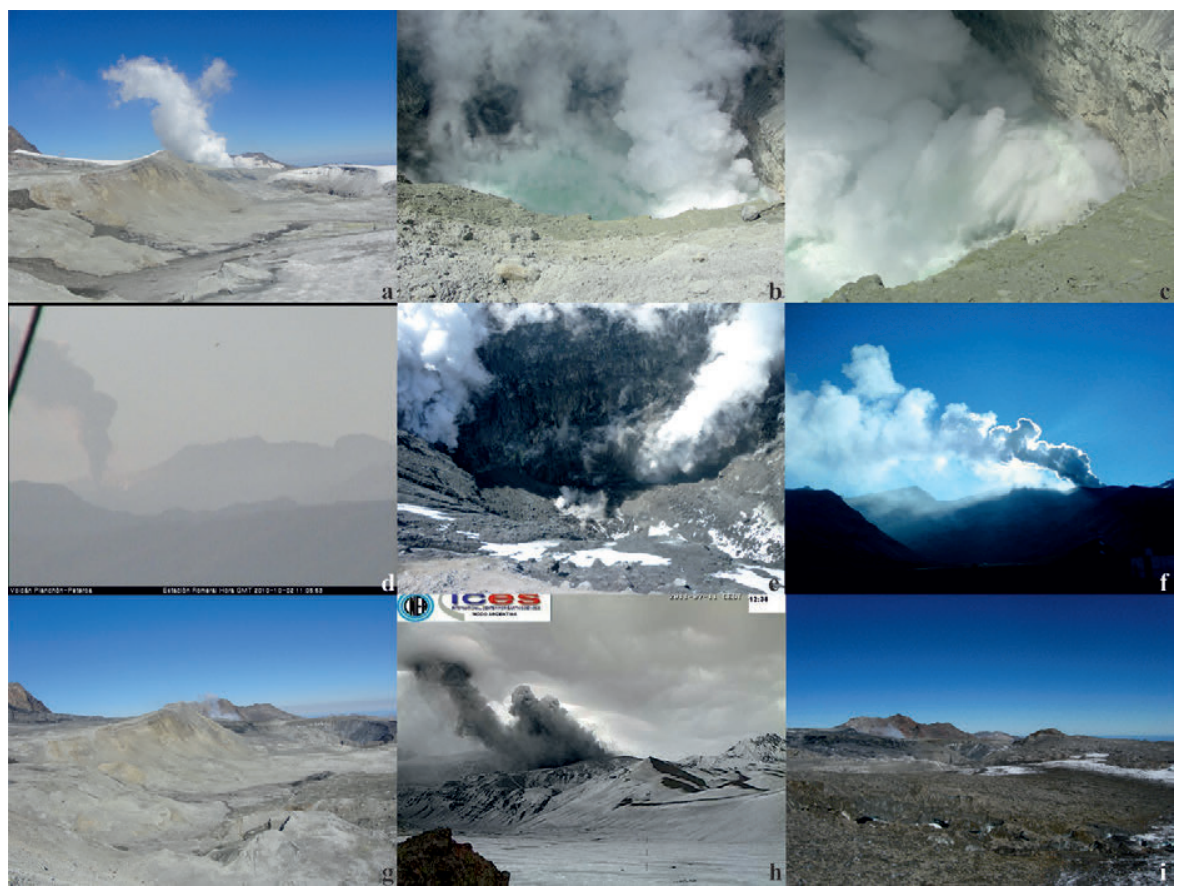

FIG. 4. a. Panoramic view from the east side of Peteroa caldera (February 2010); b. Active acid lake hosted in the crater 3, view from the W (February 2010); c. Phreatic explosion in the SW side of crater 3, view from the W (February 2010); d. Eruptive column during $2^{\text {nd }}$ October 2010, view from Romeral, $58 \mathrm{~km}$ NW Planchón-Peteroa volcano (image from surveillance camera, OVDAS-SERNAGEOMIN); e. Active crater 3 without acid lake, November 2010, view from the W (courtesy of D. Tormey); f. Eruptive column during February 2011, view from the El Azufre border office, $7 \mathrm{~km}$ E from active crater (courtesy of Gendarmería Argentina); g. Panoramic view from the east side of Peteroa caldera (March 2011); h. Eruptive column during February 2011, view from the El Azufre border office, $7 \mathrm{~km}$ E from active crater (image from surveillance camera, ICES-Argentina); i. Panoramic view from the east side of Peteroa caldera (March 2012).

and $11^{\text {th }}$ July (grey column $1,000 \mathrm{~m}$ height with fall of tephra in the SE flank of the volcano; Fig. 4h), with VEI of 2 (Table 1; Fig. 5).

\subsection{Activity after 2010-2011 eruptive period}

A permanent low height column (50-100 m) was present in the active crater after the 2010-2011 eruptive period. Low frequency seismic activity was present during August and September 2011 (186 and 274 earthquakes/month, respectively, SERNAGEOMIN, $2011 a^{1}$ and $b^{2}$ ), whereas during October 2011 the seismic frequency increases up to 675 earthquakes/ month (SERNAGEOMIN, 2011 $\mathrm{c}^{3}$ ). From November 2011 and February 2012, seismic activity was variable in a restricted range between 317 and 485 earthquakes/ month (SERNAGEOMIN, 2011 $\mathrm{d}^{4} ; 2012 \mathrm{a}^{5}, \mathrm{~b}^{6}$ and $\mathrm{c}^{7}$ ). Despite the increasing of seismic activity, eruptive activity was not detected. The last field observations in Planchón-Peteroa craters during March 2012 indicate that only a weak white column (50 m height) was present (Fig. 4i), and all craters hosted crater lakes, which is similar to the observations carried out in February 2010 (early phase 1).

${ }^{1}$ SERNAGEOMIN. 2011a. Reporte de Actividad Volcánica (RAV), Región del Maule, agosto 2011. Servicio Nacional de Geología y Minería, Informe (Unpublished), No. 25: 2 p.

${ }^{2}$ SERNAGEOMIN. 2011b. Reporte de Actividad Volcánica (RAV), Región del Maule, septiembre 2011. Servicio Nacional de Geología y Minería, Informe (Unpublished), No. 26: 2 p

${ }^{3}$ SERNAGEOMIN. 2011c. Reporte de Actividad Volcánica (RAV), Región del Maule, octubre 2011. Servicio Nacional de Geología y Minería, Informe (Unpublished), No. 27: 2 p.

${ }^{4}$ SERNAGEOMIN. 2011d. Reporte de Actividad Volcánica (RAV), Región del Maule, noviembre 2011. Servicio Nacional de Geología y Minería, Informe (Unpublished), No. 28: 2 p.

${ }^{5}$ SERNAGEOMIN. 2012a. Reporte de Actividad Volcánica (RAV), Región del Maule, diciembre 2011. Servicio Nacional de Geología y Minería, Informe (Unpublished), No. 29: 2 p.

${ }^{6}$ SERNAGEOMIN. 2012b. Reporte de Actividad Volcánica (RAV), Región del Maule, enero 2012. Servicio Nacional de Geología y Minería, Informe (Unpublished), No. 30: 2 p.

TERNAGEOMIN. 2012c. Reporte de Actividad Volcánica (RAV), Región del Maule, febrero 2012. Servicio Nacional de Geología y Minería. Informe (Unpublished), No. 31: 2 p. 


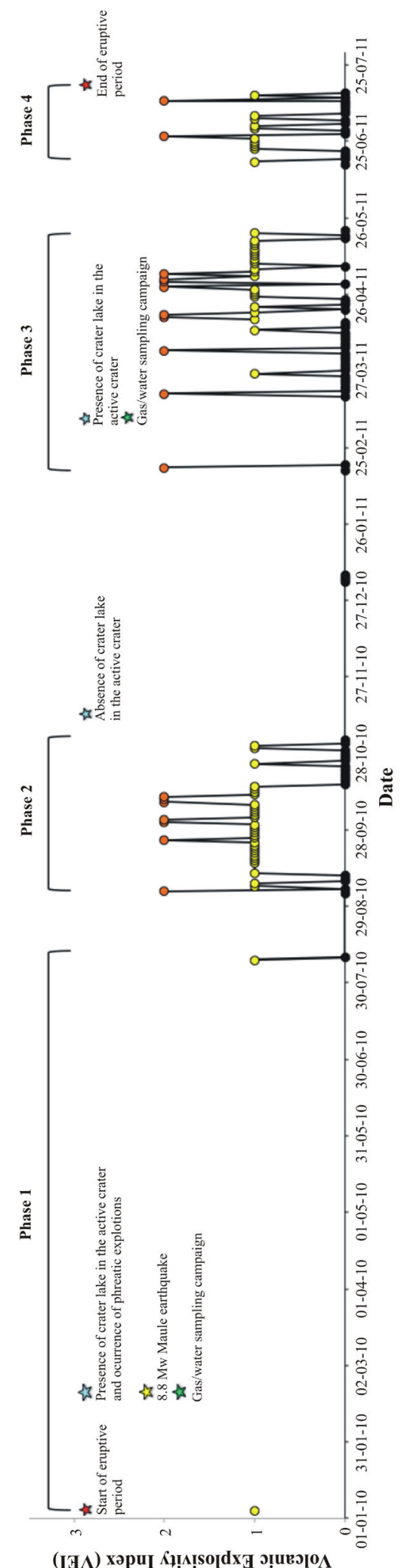

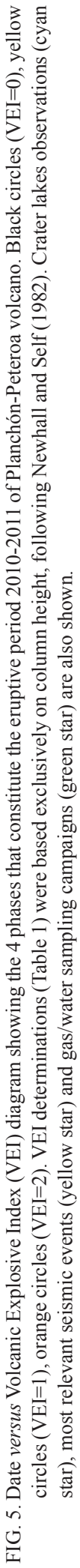

\section{Tephra fall deposit analysis}

Tephra fall deposits were observed in the whole surface of Peteroa caldera, and mainly on the NW, N, NE, E and SE flanks of Planchón-Peteroa volcano (Fig. 7a). The maximum thickness of tephra fall deposit was observed in the SSE border of crater 3 ( $4 \mathrm{~m}$ thickness; Fig. $7 \mathrm{~b}$ ), decreasing progressively to $1 \mathrm{~cm}$ thickness $\sim 20 \mathrm{~km} \mathrm{SE}$ and $\sim 7 \mathrm{~km} \mathrm{NW}$ from the active crater, and $0.5 \mathrm{~cm}$ thickness $\sim 30 \mathrm{~km} \mathrm{SE}$ and $\sim 8 \mathrm{~km}$ NW from the active crater. Maximum area covered by tephra fall deposit extended up to $1,265 \mathrm{~km}^{2}$ during $10^{\text {th }}$ October 2010 (Table 1).

Several sites located on the NE, E and SE flanks of volcano were sampled during phases 2 and 3. Tephra fall deposits of phases 2 and 3 are almost identical, and are characterized by a size range between $-1 \phi(2$ $\mathrm{mm}$ ) and $>3.75 \phi(0.075 \mathrm{~mm})$ (Table 2$)$, corresponding mostly to coarse ash. Tephra fall deposits are constituted by lithics fragments (40-80\% vol.), quartz (5-50\% vol.) and plagioclase (10-25\% vol.) crystals. Lithics fragments $(>65 \%$ vol.) dominate in sizes $<1.25 \phi$ $(0.425 \mathrm{~mm})$, whereas for sizes between 1.25 and $3.75 \phi(0.425$ and $0.075 \mathrm{~mm})$, lithics fragments and quartz crystals are present in similar quantities ( $\sim 5 \%$ vol.). The smallest fraction, corresponding to $>3.75 \phi(0.075 \mathrm{~mm})$, is dominated by quartz crystals ( $80 \%$ vol.) and scarce lithics fragments and plagioclase crystals (10 and 5\% vol., respectively). Lithics fragments are constituted mainly by three different types of dark grey to black fragments, corresponding to porphyry lava, glassy porphyritic lava and vesicular lava fragments. Additionally, some lithic fragments are partially oxidized, appearing as reddish brown fragments. Oxide $\mathrm{Fe}$ minerals, and less frequently $\mathrm{Cu}$ minerals, were occasionally observed as single fragments. Cu minerals have been commonly observed in crater lake environments (e.g., Copahue volcano-Chile, Keli Mutu volcanoIndonesia; Pasternack and Varekamp, 1994; Varekamp and Ouimette, 2001). Granulometric (Table 2) and statistical analysis indicate that tephra fall deposits are multimodal (3 modes; 1, 2 and $3.75 \phi$, respectively), suggesting that Planchón-Peteroa eruptions corresponding to highly explosive events, probably related to phreatic processes (Wohletz, 1983). Walker (1971) used statistical parameters such as the graphical median (Md) and graphical standard deviation $\left(\sigma_{\mathrm{G}}\right)$ to determine the type of deposit. In this case, $\mathrm{Md} 0.11 \phi$ and $\sigma_{\mathrm{G}} 0.35 \phi$ suggest that Planchón- 


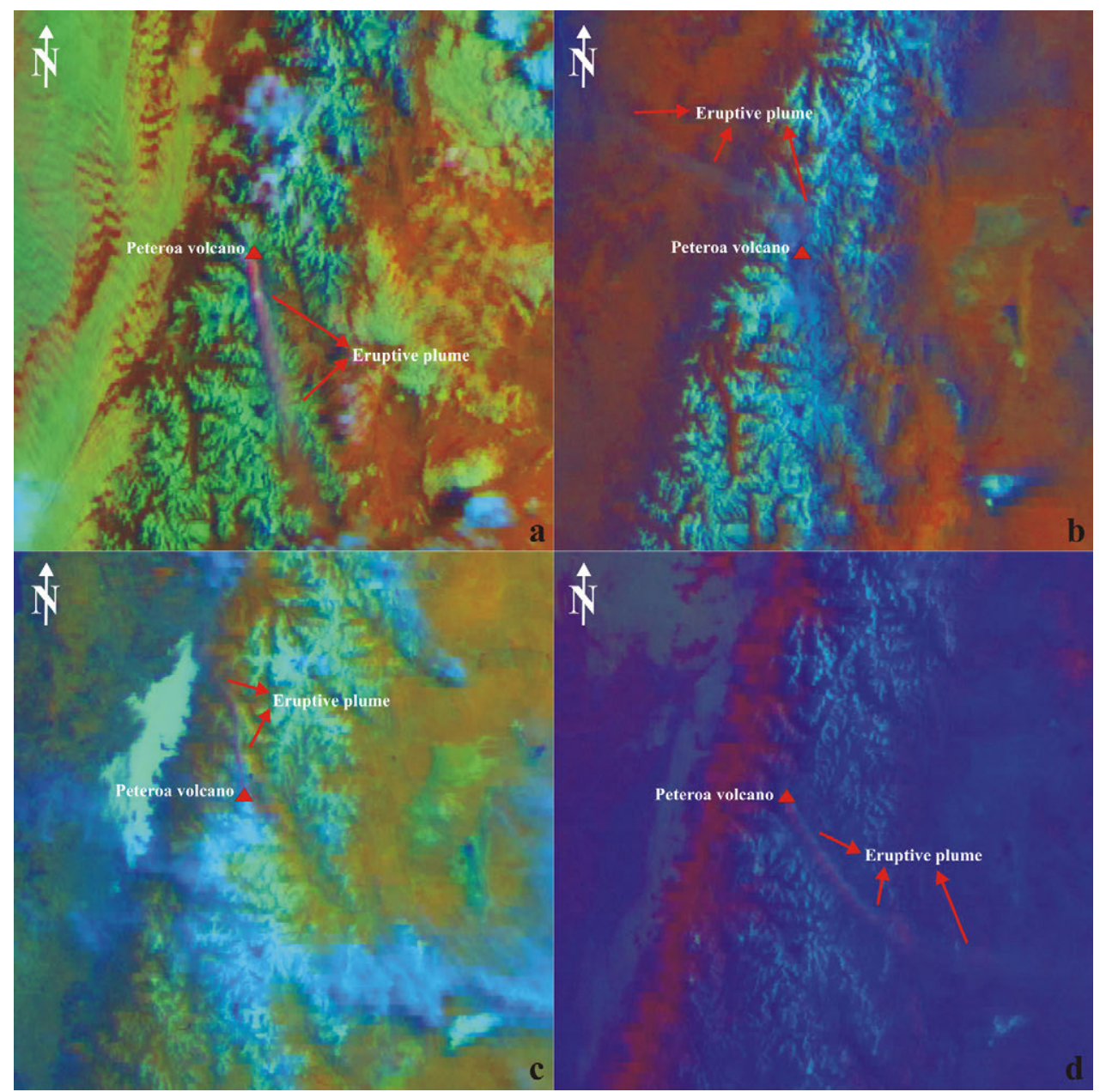

FIG. 6. GOES satellite RGB images of: a. 26 September 2010; b. 2 October 2010; c. 4 May 2011 and d. 6 May 2011, showing the displacement of eruptive plumes. Position of Planchón-Peteroa volcano (red triangle) is also shown.

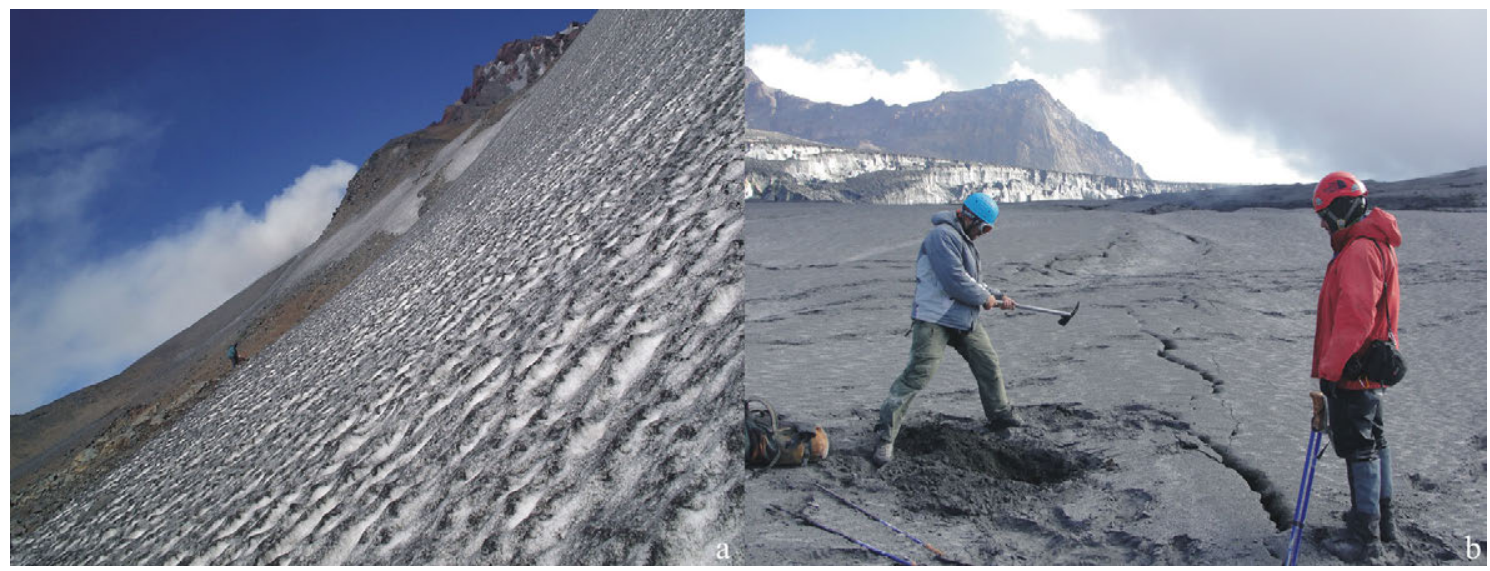

FIG. 7. Tephra fall deposit on the a) NE flank of Planchón-Peteroa volcano (note a person in the middle-left side of the photo as scale) and b) SE border of crater 3 . 
TABLE 2. PHYSICAL PARAMETERS OF TEPHRA FALL DEPOSIT SIEVED.

\begin{tabular}{|c|c|c|c|c|c|c|}
\hline Sieve No. & Diameter (mm) & phi size & Weight (grs) & Weight (\%) & $\begin{array}{c}\text { Cumulative weight } \\
\text { (grs) }\end{array}$ & $\begin{array}{c}\text { Cumulative weight } \\
\text { (\%) }\end{array}$ \\
\hline 1 & 2 & -1 & 7.84 & 0.98 & 7.84 & 0.98 \\
\hline 2 & 1 & 0 & 68.88 & 8.65 & 76.72 & 9.64 \\
\hline 3 & 0.5 & 1 & 105.06 & 13.2 & 181.78 & 22.83 \\
\hline 4 & 0.425 & 1.25 & 28.58 & 3.59 & 210.36 & 26.42 \\
\hline 5 & 0.25 & 2 & 79.28 & 9.96 & 289.64 & 36.38 \\
\hline 6 & 0.18 & 2.5 & 39.73 & 4.99 & 329.37 & 41.37 \\
\hline 7 & 0.15 & 2.75 & 21.28 & 2.67 & 350.65 & 44.04 \\
\hline 8 & 0.075 & 3.75 & 112.82 & 14.17 & 463.47 & 58.21 \\
\hline
\end{tabular}

Diameter (in mm) and phi $(\phi)$ size of particles, weight (in grs and percentage) and cumulative weight (in grs. and percentage) are shown.

Peteroa tephra deposit is related to pyroclastic fall process (Fig. 8), similar to what is suggested by the field measurements and direct observations.

\section{Fluid geochemistry analysis}

\subsection{Water geochemistry from crater lakes}

Water geochemistry analysis from the crater lakes 1, 2 and 4 indicate that all the crater lakes were developed under similar physical and chemical conditions (Benavente et al., 2013; Benavente, 2015; Tassi et al., in press; Table 3). pH varies between 1.49 and 2.91, while temperature $(\mathrm{T})$ is more variable, being colder in crater $1\left(7.4{ }^{\circ} \mathrm{C}\right)$, followed by crater $4\left(17.1^{\circ} \mathrm{C}\right)$, and the hottest is the lake hosted in the crater 2 , which presented a $\mathrm{T}$ of $43.2^{\circ} \mathrm{C}$ on March 2011 and $30.6^{\circ} \mathrm{C}$ on March 2012. The total of dissolved solids (TDS) varies in a limited range from 3,247 to 4,159 mg/l (Table 3). Sulphate is the principal anion in all crater lake waters (Fig. 9a), and its origin seems to be related to the condensation of steam emitted from the fumarolic vents located in the border and bottom of crater lakes and oxidation of $\mathrm{SO}_{2}$ and $\mathrm{H}_{2} \mathrm{~S}$ in the near-surface water. Chlorine is the second anion in order of abundance, especially in the crater 1 where its contents reach up to $662 \mathrm{mg} / \mathrm{l}$ (Table 3), which seems to be related to the absorption of $\mathrm{HCl}$ in the near-surface water. Bicarbonate is absent in all the crater lakes, which is consistent with the low $\mathrm{pH}$ values of the lakes $(<3)$. The cations with the highest concentrations correspond to $\mathrm{Ca}^{2+}(182$ to $523 \mathrm{mg} / \mathrm{l}$ ) and $\mathrm{Mg}^{2+}$ (95 to $377 \mathrm{mg} / \mathrm{l}$ ) (Table 3). As is shown in figure $9 \mathrm{~b}$, crater lake samples plot close to the Global Meteoric Water Line (GMWL), indicating that rain and melting of glaciers hosted in Peteroa caldera are the principal water sources for the crater lakes. The subtle $\delta^{18} \mathrm{O}$-shifting to more positive values respect to GMWL depicted by the crater lake samples in figure 9b may be explained as the result of a limited mixing with condensated water vapour from the fumarolic vents surrounding the crater. Even though waters from the lake hosted in crater 2 where sampled in two different periods, no major changes in the physicochemical parameters were observed.

\subsection{Gas geochemistry from fumaroles}

The gas composition from fumaroles sampled during February 2010 (early phase 1) indicates that fumarolic fluids are enriched in $\mathrm{H}_{2} \mathrm{~S}$ and $\mathrm{CH}_{4}$ (Table 4; Fig. 10a, b and c), which are typical gas species related to hydrothermal environments (e.g., Giggenbach, 1987, 1996). According to figure 10a, b and $\mathrm{c}$, a limited contribution from atmospheric (i.e., $\mathrm{Ar}$ addition) and magmatic sources $\left(\mathrm{SO}_{2}, \mathrm{HCl}\right.$ and HF; Giggenbach, 1996) are also observed in these gas samples. Fumaroles sampled on March 2011 (eruptive phase 3 ) evidence a limited increasing in the contents of acid species ( $\mathrm{SO}_{2}, \mathrm{HCl}$ and $\mathrm{HF}$ ) and a decrease of atmospheric species like $\mathrm{Ar}$ and $\mathrm{O}_{2}$ (Table 4). This seems to be related to an increase (but limited) of the fluids released from a magmatic source as these samples were taken during the $3^{\text {rd }}$ 


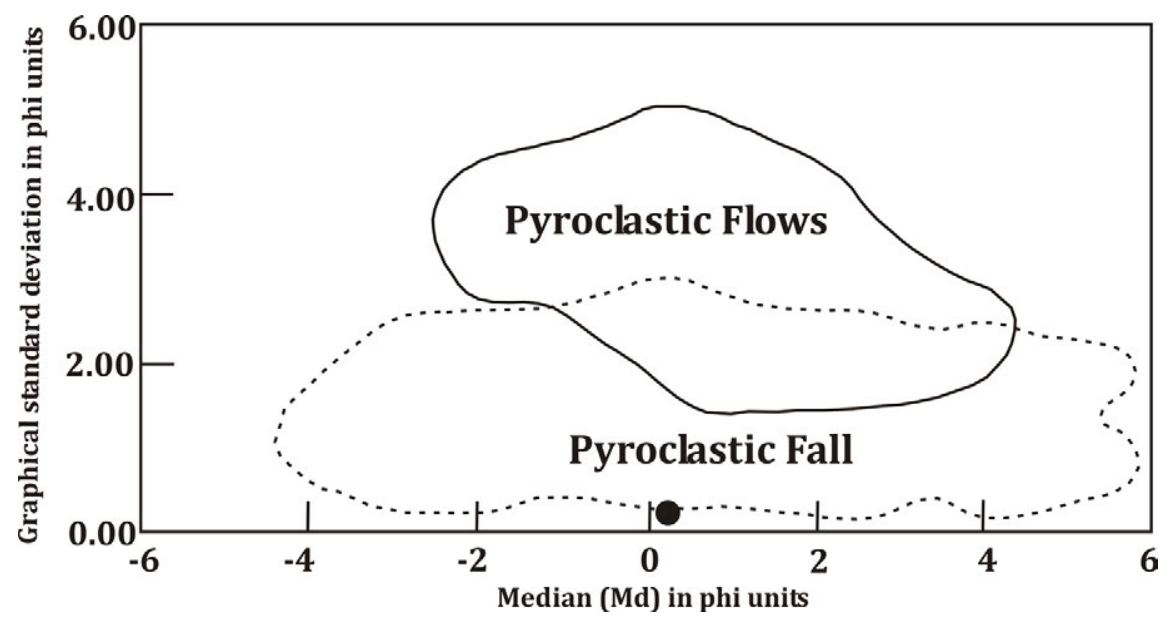

FIG. 8. Empiric diagram for granulometric classification of pyroclastic deposits (after Walker, 1971). Black dot indicate the graphical median $(\mathrm{Md})$ and graphical standard deviation $\left(\sigma_{\mathrm{G}}\right)$ values for Planchón-Peteroa pyroclastic fall deposit.

TABLE 3. OUTLET TEMPERATURES $\left(\right.$ IN $\left.{ }^{\circ} \mathrm{C}\right)$, pH, CHEMICAL COMPOSITION AND $\delta^{18} \mathrm{O}$ AND $\delta \mathrm{D}(\% \mathrm{~V}-\mathrm{SMOW})$ VALUES OF THE WATERS FROM CRATER LAKES HOSTED IN PLANCHÓN-PETEROA VOLCANO.

\begin{tabular}{|c|c|c|c|c|c|c|c|c|c|c|c|c|c|c|c|c|c|c|}
\hline Sample & Type & Date & $\begin{array}{c}\mathbf{T} \\
\left({ }^{\circ} \mathbf{C}\right)\end{array}$ & pH & $\mathrm{HCO}_{3}$ & $\mathrm{Cl}$ & $\mathrm{SO}_{4}$ & $\mathrm{Na}$ & $\mathbf{K}$ & $\mathrm{Ca}$ & Mg & $\mathrm{NO}_{3}$ & F & $\mathrm{Br}$ & $\mathbf{N H}_{4}$ & TDS & $\delta \mathbf{D}$ & $\delta^{18} \mathbf{O}$ \\
\hline Peteroa 5 & $\begin{array}{l}\text { Crater } \\
\text { lake }\end{array}$ & 2011 & 43.2 & 1.49 & n.d. & 44 & 3,288 & 82 & 133 & 223 & 140 & 2.03 & 5.2 & 0.06 & 9.98 & 3,927 & -74 & -9.5 \\
\hline Peteroa 5 & $\begin{array}{l}\text { Crater } \\
\text { lake }\end{array}$ & 2012 & 30.6 & 1.50 & n.d. & 92 & 2,794 & 63 & 16 & 182 & 95 & 0.81 & 2.5 & 0.52 & n.d. & 3,247 & n.a. & n.a. \\
\hline Peteroa 9 & $\begin{array}{l}\text { Crater } \\
\text { lake }\end{array}$ & 2011 & 19.1 & 2.73 & n.d. & 197 & 3,018 & 182 & 42 & 326 & 377 & 1.00 & 9.9 & 0.77 & 4.92 & 4,159 & -80 & -9.9 \\
\hline $\begin{array}{l}\text { Peteroa } \\
10\end{array}$ & $\begin{array}{l}\text { Crater } \\
\text { lake }\end{array}$ & 2011 & 7.4 & 2.91 & n.d. & 662 & 2,001 & 145 & 34 & 523 & 182 & 1.00 & 39.2 & 1.43 & 2.63 & 3,591 & -80 & -10.1 \\
\hline
\end{tabular}

Concentrations of solutes are in mg/L; n.d.: not detected; n.a.: not analyzed. Data from Saltori (2014), Benavente (2015) and Tassi et al., in press.

eruptive phase (Fig. 10a, b and c). This magmatic input can also be observed in figure $9 \mathrm{~b}$ where the $\delta \mathrm{D}$ - and $\delta^{18} \mathrm{O}-\mathrm{SMOW}$ values of the fumarolic samples suggest a mixing between a meteoric (mostly corresponding to rain water at 4,000 $\mathrm{m}$ a.s.1.) and an "andesitic" source (e.g., Tarán et al., 1989; Giggenbach, 1992). Even though fumarolic fluids at March 2011 present different evidences indicating a magmatic input, the chemical composition of the fluids are still dominated by components originated in a typical hydrothermal environment (e.g., $\mathrm{H}_{2} \mathrm{~S}$, $\mathrm{CH}_{4}$; Table 4).

The most dramatic physico-chemical change occurred after the 2010-2011 eruptive period (March 2012), where a strong increase of the magmatic acid species content occurred. $\mathrm{SO}_{2}$ abundance increased by three folds with respect to the samples taken on March 2011, whereas $\mathrm{HCl}$ and $\mathrm{HF}$ contents increased by two order of magnitude (Table 4). Despite of divergent changes in the concentration of the hydrothermal species with respect to the samples taken in 2010 and 2011 (i.e., decreases of $\mathrm{CH}_{4}$ and increases of $\mathrm{H}_{2} \mathrm{~S}$ ), the relative proportions of $\mathrm{SO}_{2}$, as well as the contents of the other acid species on March 2012 suggest that fumaroles were strongly dominated by magmatic source fluids on March 2012 (Fig. 10 a and b). Contrary to what intuition would indicate, samples collected during the "magmatic" stage (March 2012), as well as the water samples of the crater lakes and the bubbling pools plot along the GMWL in figure $9 \mathrm{~b}$. We argue that the $\delta \mathrm{D}$ and $\delta^{18} \mathrm{O}$ temporal variations at the crater fumaroles between March 2011 and 2012 (Fig. 9b) 

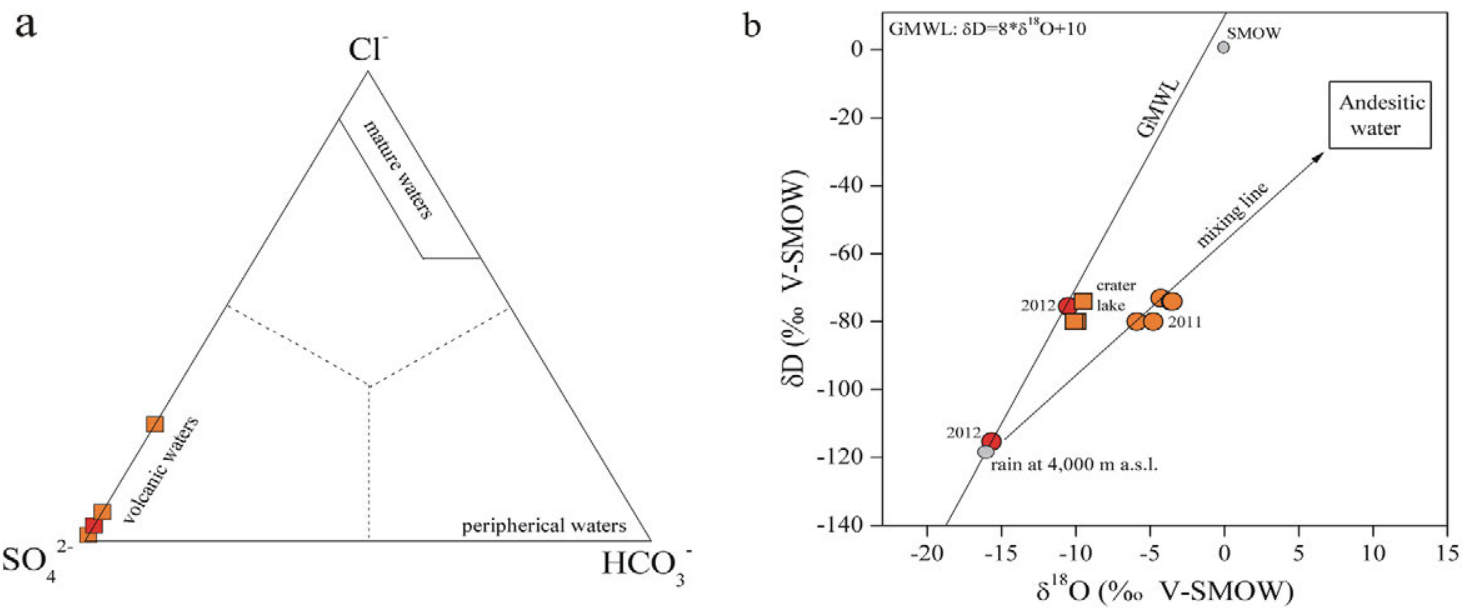

FIG. 9. a. $\mathrm{HCO}_{3}^{-}-\mathrm{Cl}^{-}-\mathrm{SO}_{4}{ }^{2-}$ diagram for water samples from crater lakes; b. $\delta^{18} \mathrm{O}-\delta \mathrm{D}$ diagram for water samples from crater lakes (filled squares) and gas condensates from fumarolic emisions (filled circles). The "andesitic water" field (Taran et al., 1989; Giggenbach, 1992), Global Meteoric Water Line (GMWL), Standard Mean Ocean Water (SMOW) field and local precipitation isotope composition are also shown. Orange symbols for 2011 and red symbols for 2012 samples.

are related to an episode of minimum and maximum availability of rain water and/or melted glacial water in the shallow aquifer hosted at Planchón-Peteroa volcano. Field observations of the crater from where the gas condensates were taken in March 2011, which is also near to the sampling points in March 2012 (crater 2), indicate the presence of an acid lake only in March 2012, supporting this hypothesis.

\subsection{Geothermometry from gaseous phase}

Even though no major changes in outlet temperatures at the fumarolic vents were observed during and after the 2010-2011 eruptive period (Table 4), the chemical changes detected in the previous revision (section 7.2) can be an indicator of variations in the physicochemical conditions of fluids at depth (e.g., temperature, pressure, redox), as has been observed in other active volcanoes (e.g., Fischer et al., 1997; Vaselli et al., 2010; Tassi et al., 2013). Accordingly, a thermodynamic review is done with the objective to detect possible variations in the physicochemical conditions of fluids at depth.

According to Giggenbach (1993), theoretical temperatures of magmatic-hydrothermal fluids in the $\mathrm{CH}_{4}-\mathrm{CO}_{2}-\mathrm{H}_{2}$ system can be calculated by the following reaction:

$$
\mathrm{CH}_{4}+2 \mathrm{H}_{2} \mathrm{O} \leftrightarrow 4 \mathrm{H}_{2}+\mathrm{CO}_{2}
$$

The temperature dependence of the equilibrium constant of reaction (1) in the equilibrated vapour and liquid is given by:

$\log \left(\mathrm{X}_{\mathrm{CH}_{4}} / \mathrm{X}_{\mathrm{CO}_{2}}\right)_{\mathrm{v}}=4 \mathrm{R}_{\mathrm{H}}+5181 / \mathrm{T}(\mathrm{K})$

$\log \left(\mathrm{X}_{\mathrm{CH}_{4}} / \mathrm{X}_{\mathrm{CO}_{2}}\right)_{1}=4 \mathrm{R}_{\mathrm{H}}+5181 / \mathrm{T}+\log \left(\mathrm{B}_{\mathrm{CO}_{2}}\right)-\log \left(\mathrm{B}_{\mathrm{CH}_{4}}\right)$

where $\mathrm{R}_{\mathrm{H}}=\log \left(\mathrm{X}_{\mathrm{H}_{2}} / \mathrm{X}_{\mathrm{H}_{2} \mathrm{O}}\right), \log \left(\mathrm{X}_{\mathrm{H}_{2} \mathrm{O}}\right) \approx \log \left(\mathrm{f}_{\mathrm{H}_{2} \mathrm{O}}\right)$ $=4.9-(1820 / \mathrm{T})$, $\mathrm{T}$ is in $\mathrm{K}$, and $\mathrm{B}_{\mathrm{CO}_{2}}$ and $\mathrm{B}_{\mathrm{CH}_{4}}$ are the vapour/liquid distribution coefficients of $\mathrm{CO}_{2}$ and $\mathrm{CH}_{4}$, respectively (Giggenbach, 1987, 1991). The $\mathrm{B}_{\mathrm{CO}_{2}}$ and $\mathrm{B}_{\mathrm{CH}_{4}}$ values at different temperatures are calculated on the polynomial equations reported by Sepúlveda et al. (2007). Assuming that Ar in magmatic-hydrothermal fluids have an atmospheric origin and is controlled by the equilibrium between the atmosphere and air-saturated water $\left(\log \left(r_{\mathrm{Ar}}\right)=-6.52\right.$, where $r_{\mathrm{Ar}}$ is the Ar mol-ratio), Giggenbach (1993) suggested that at equilibrium, $\mathrm{H}_{2}$ concentrations in the equilibrated vapour and liquid were related to $R_{H}$ by the following equations:

$$
\begin{aligned}
& \log \left(\mathrm{X}_{\mathrm{H}_{2}} / \mathrm{X}_{\mathrm{Ar}}\right)_{\mathrm{v}}=\mathrm{R}_{\mathrm{H}}-\log \left(r_{\mathrm{Ar}}\right) \\
& \log \left(\mathrm{X}_{\mathrm{H}_{2}} / \mathrm{X}_{\mathrm{Ar}}\right)_{1}=\mathrm{R}_{\mathrm{H}}-\log \left(r_{\mathrm{Ar}}\right)-\log \left(\mathrm{B}_{\mathrm{H}_{2}}\right)
\end{aligned}
$$

where the vapour/liquid distribution coefficients $\mathrm{B}_{\mathrm{H}_{2}}$ was calculated from the same data sources used 
TABLE 4. OUTLET TEMPERATURES (IN $\left.{ }^{\circ} \mathrm{C}\right)$, CHEMICAL COMPOSITION AND $\delta^{18}$ O AND $\delta$ D ISOTOPE RATIOS IN CONDENSATES OF THE FUMAROLIC DISCHARGES FROM PLANCHÓN-PETEROA VOLCANO.

\begin{tabular}{|c|c|c|c|c|c|c|c|c|c|c|c|c|c|c|c|c|c|}
\hline Sample & Type & Date & $\mathbf{T}\left({ }^{\circ} \mathrm{C}\right)$ & $\mathrm{CO}_{2}$ & $\mathrm{HCl}$ & HF & $\mathrm{SO}_{2}$ & $\mathrm{H}_{2} \mathrm{~S}$ & $\mathbf{N}_{2}$ & $\mathrm{CH}_{4}$ & Ar & $\mathrm{O}_{2}$ & $\mathbf{H}_{2}$ & $\mathrm{He}$ & $\mathrm{CO}$ & $\delta \mathbf{D}$ & $\delta^{18} \mathrm{O}$ \\
\hline Peteroa 1 & Fumarole & 2010 & 91.8 & 978 & 0.246 & 0.011 & 0.115 & 19.3 & 1.56 & 0.237 & 0.026 & 0.056 & 0.364 & 0.0032 & 0.0026 & n.a. & n.a. \\
\hline Peteroa 1 & Fumarole & 2011 & 87.7 & 975 & 0.374 & 0.009 & 0.550 & 21.0 & 2.62 & 0.132 & 0.004 & 0.006 & 0.245 & 0.0014 & 0.0150 & -80 & -5.9 \\
\hline Peteroa 1 & Fumarole & 2012 & 87.8 & 363 & 14.1 & 1.3 & 466 & 151 & 7.55 & 0.009 & 0.016 & 0.006 & 0.612 & 0.0025 & 0.0008 & n.a. & n.a. \\
\hline Peteroa 2 & Fumarole & 2010 & 102.1 & 961 & 0.189 & 0.021 & 0.168 & 35.4 & 2.75 & 0.286 & 0.024 & 0.049 & 0.384 & 0.0028 & 0.0031 & n.a. & n.a. \\
\hline Peteroa 3 & Fumarole & 2011 & 88.4 & 979 & 0.384 & 0.012 & 0.509 & 18.0 & 2.26 & 0.098 & 0.002 & 0.005 & 0.209 & 0.0012 & 0.0092 & -73 & -4.3 \\
\hline Peteroa 3 & Fumarole & 2012 & 89.4 & 349 & 12.9 & 1.3 & 474 & 156 & 6.54 & 0.012 & 0.018 & 0.005 & 0.533 & 0.0034 & 0.0006 & n.a. & n.a. \\
\hline Peteroa 4 & Fumarole & 2011 & 88.4 & 978 & 0.318 & 0.012 & 0.402 & 18.1 & 2.54 & 0.109 & 0.004 & 0.028 & 0.177 & 0.0013 & 0.0089 & -74 & -3.6 \\
\hline Peteroa 5 & Fumarole & 2011 & 43.2 & 986 & 0.235 & 0.008 & 0.308 & 10.8 & 2.50 & 0.122 & 0.008 & 0.028 & 0.131 & 0.0015 & 0.0050 & -74 & -3.5 \\
\hline Peteroa 6 & Fumarole & 2011 & 87.9 & 978 & 0.314 & 0.010 & 0.411 & 18.9 & 2.38 & 0.102 & 0.005 & 0.007 & 0.231 & 0.0015 & 0.0147 & -80 & -4.8 \\
\hline Peteroa 7 & Fumarole & 2011 & 88.3 & 980 & 0.383 & 0.011 & 0.539 & 16.2 & 2.35 & 0.091 & 0.004 & 0.001 & 0.279 & 0.0022 & 0.0117 & n.a. & n.a. \\
\hline Peteroa 7 & Fumarole & 2012 & 87.9 & 343 & 13.3 & 1.2 & 475 & 160 & 6.89 & 0.008 & 0.011 & 0.007 & 0.478 & 0.0043 & 0.0019 & -75.5 & -10.51 \\
\hline Peteroa 8 & Fumarole & 2012 & 87.9 & 351 & 12.1 & 1.2 & 482 & 146 & 7.13 & 0.011 & 0.014 & 0.003 & 0.511 & 0.0032 & 0.0022 & -115.3 & -15.67 \\
\hline
\end{tabular}

Gas contents are in mmol/mol; n.a.: not analyzed. Data from Saltori (2014), Benavente (2015) and Tassi et al., in press. 

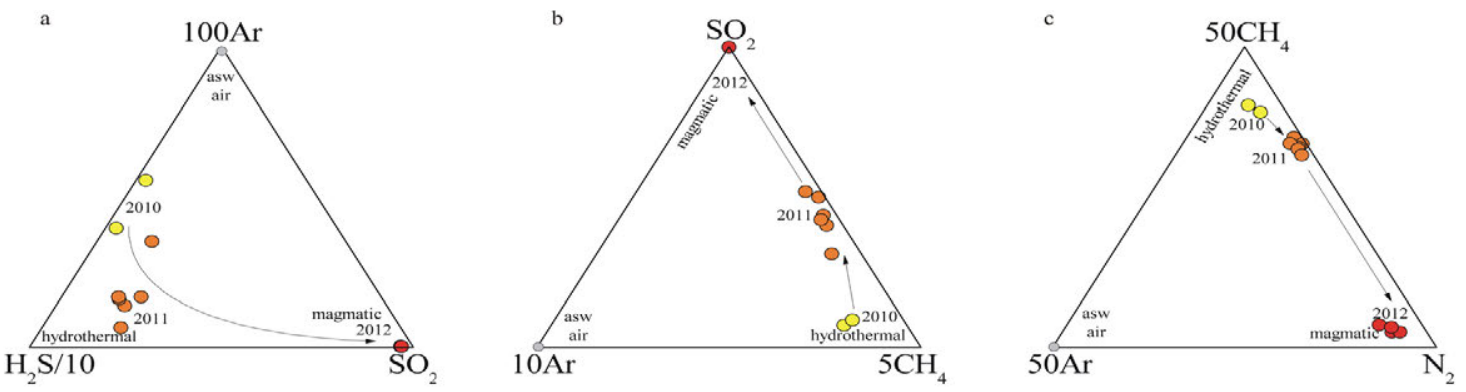

FIG. 10. a. $\left(\mathrm{SO}_{2}\right)-100 *(\mathrm{Ar})-\left(\mathrm{H}_{2} \mathrm{~S}\right) / 10$; b. $\left(\mathrm{CH}_{4}\right)-\mathrm{SO}_{2}-10 * \mathrm{Ar}$; c. $\left(\mathrm{N}_{2}\right)-50 *\left(\mathrm{CH}_{4}\right)-50$ Ar ternary diagrams for fumaroles of Planchón-Peteroa volcano. Fumaroles collected in 2010 (yellow filled circles), 2011 (orange filled circles) and 2012 (red filled circles).

for those of $\mathrm{CO}_{2}$ and $\mathrm{CH}_{4}$. In figure $11 \mathrm{a}$ the $\log \left(\mathrm{X}_{\mathrm{H}_{2}} /\right.$ $\left.\mathrm{X}_{\mathrm{Ar}^{*}}\right)$ versus $\log \left(\mathrm{X}_{\mathrm{CH} 4} / \mathrm{X}_{\mathrm{CO}_{2}}\right)$ grid is constructed on the basis of equations $2,3,4$ and 5 at different $\mathrm{R}_{\mathrm{H}}$ and temperatures. The Ar* values, used in figure 11a instead of the Ar molar concentrations, were calculated as follows:

$\operatorname{Ar} *=\operatorname{Ar}-\left(\mathrm{O}_{2} / 22\right)$

The $\mathrm{O}_{2} / 22$ values correspond to the amounts of $\mathrm{Ar}$ added by atmospheric contamination, because $\mathrm{O}_{2}$ is completely absent in pristine hydrothermal fluids and only occurs as the result of shallow level atmospheric contamination (Giggenbach, 1993). The gases collected at the crater during the complete eruptive period plot along the liquid equilibrium curve at $R_{H}$ values near -3.1, with temperatures increasing from $\sim 290$ ${ }^{\circ} \mathrm{C}$ for 2010 samples (eruptive phase 1) to $\sim 330{ }^{\circ} \mathrm{C}$ for 2011 samples (eruptive phase 3) (Fig. 11a). Such redox conditions are lower than those dictated by the $\mathrm{FeO} / \mathrm{FeO}_{1.5}$ "rock" buffer $\left(\mathrm{R}_{\mathrm{H}}=-2.8\right)$ and they can be ascribed to inputs of oxidizing deep magmatic-related fluids into the shallow hydrothermal environment, which agree with the presence of $\mathrm{SO}_{2}$ in the crater gases during the 2010-2011 unrest period (Table 4; Giggenbach, 1987). The distribution of the gases sampled after eruptive period (2012) shows higher oxidizing conditions $\left(\mathrm{R}_{\mathrm{H}} \approx-3.25\right)$ with respect to the prior period, although there is no significant increment of the equilibrium temperature respect to 2011 samples (Fig. 11a). The evolution of equilibrium conditions indicated by the $\mathrm{CO}_{2}-$ $\mathrm{CH}_{4}-\mathrm{H}_{2}-\mathrm{Ar}$ systems is consistent with the changes from a magmatic-hydrothermal (in 2010-2011) to a "magmatic" stage (in 2012).

Chemical equilibria in the $\mathrm{H}_{2}-\mathrm{CO}_{2}-\mathrm{CO}-\mathrm{CH}_{4}-\mathrm{H}_{2} \mathrm{O}$ system can also be considered for geothermometric evaluation of the Planchón-Peteroa fumarolic fluids (Chiodini and Marini, 1998). The equilibrium conditions can be adequately described by the following two redox-independent reactions (Chiodini and Marini, 1998):

$\mathrm{CO}_{2}+\mathrm{H}_{2} \leftrightarrow \mathrm{H}_{2} \mathrm{O}+\mathrm{CO}$

$\mathrm{CH}_{4}+3 \mathrm{CO}_{2} \leftrightarrow 4 \mathrm{CO}+2 \mathrm{H}_{2} \mathrm{O}$

The analytical values of the thermodynamic constants of equations (7) and (8), i.e., $\log \left(\mathrm{H}_{2} \mathrm{O} /\right.$ $\left.\mathrm{H}_{2}\right)+\log \left(\mathrm{CO} / \mathrm{CO}_{2}\right)$ and $3 \log \left(\mathrm{CO} / \mathrm{CO}_{2}\right)+\log \left(\mathrm{CO} / \mathrm{CH}_{4}\right)$, are controlled by the temperature of an original saturate liquid phase $\left(\mathrm{T}_{\mathrm{o}}\right)$ and either adiabatic singlestep steam separation temperature $\left(\mathrm{T}_{\mathrm{s}}\right.$; Fig. 11b). As shown in figure $11 \mathrm{~b}$, despite some spread, fumarolic samples of Planchón-Peteroa volcano plot within the vapour+liquid field. The evolution of the equilibrium conditions in figure $11 \mathrm{~b}$ indicates the occurrence of an increasing "energetic" boiling of the magmatic-hydrothermal reservoir during the unrest episode (2010-2011). In February 2010 vapor phase was separated from a liquid zone $\left(\mathrm{T}_{\mathrm{o}} \sim 275^{\circ} \mathrm{C}\right)$ at a temperature $\mathrm{T}_{\mathrm{s}}>200^{\circ} \mathrm{C}$ and a pressure $>16$ bar; whereas in March 2011 fumarolic gases plot near the liquid line at $\mathrm{T}_{\mathrm{o}} \sim 350{ }^{\circ} \mathrm{C}$ and a $\mathrm{T}_{\mathrm{s}}<150{ }^{\circ} \mathrm{C}$. The relatively low $\mathrm{H}_{2}$ and $\mathrm{CO}$ contents of sample Pet-5 in March 2011 (Table 4), which plots far from the gases collected in 2011, are likely related to the extremely low flux of this fumarole $\left(\mathrm{T} \sim 43^{\circ} \mathrm{C}\right.$; Table 4$)$, which favors oxidation of these highly reactive gas compounds at shallow depth. Fumarolic gases collected during the "magmatic" stage (2012), on the other hand, plot close to a $\mathrm{T}_{\mathrm{o}}$ and $\mathrm{T}_{\mathrm{s}}$ up to $325^{\circ} \mathrm{C}$ and $275^{\circ} \mathrm{C}$, respectively (Fig. $11 \mathrm{~b}$ ), evidencing a steam separation at higher pressures ( $\sim 60 \mathrm{bar})$. 


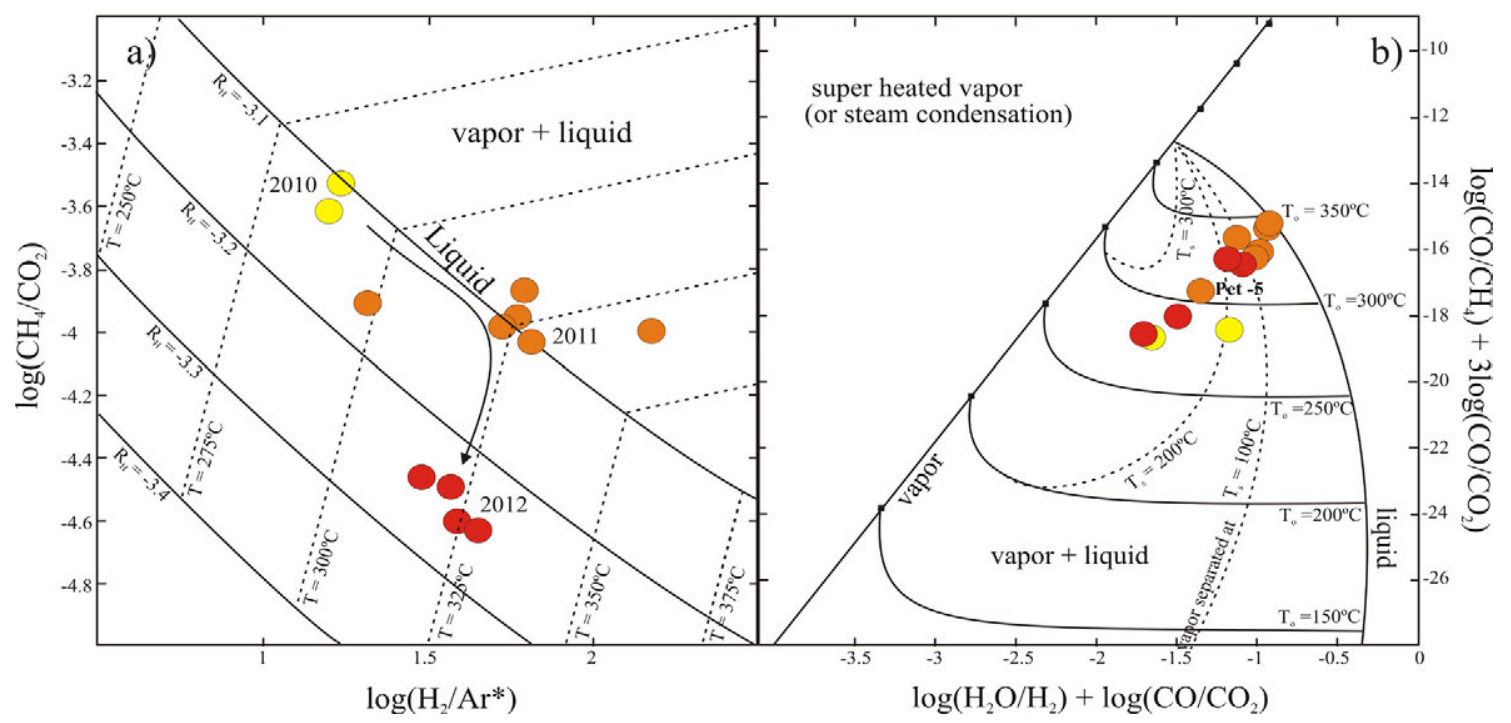

FIG. 11. a. $\log \left(\mathrm{H}_{2} / \mathrm{Ar} *\right)$ versus $\log \left(\mathrm{CH}_{4} / \mathrm{CO}_{2}\right)$ diagram for the thermal discharges sampled at Planchón-Peteroa volcano. Ar*=Ar- $\mathrm{O}_{2} / 22$; $\mathrm{R}_{\mathrm{H}}=\log \left(\mathrm{H}_{2} / \mathrm{H}_{2} \mathrm{O}\right)$ (Giggenbach, 1987). b. $3 \log \left(\mathrm{CO} / \mathrm{CO}_{2}\right)+\log \left(\mathrm{CO} / \mathrm{CH}_{4}\right)$ versus $\log \left(\mathrm{H}_{2} \mathrm{O} / \mathrm{H}_{2}\right)+\log \left(\mathrm{CO} / \mathrm{CO}_{2}\right)$ binary diagram for the Planchón-Peteroa volcano fumaroles after Chiodini and Marini (1998). Theoretical compositions were calculated considering gas solubility and thermodynamic data for pure water. Symbols as figure 10.

\section{Discussion}

The renewing of Planchón-Peteroa volcano activity (phase 1) was characterized by an almost permanent emission of a $\sim 200 \mathrm{~m}$ gas/steam column during 7 months as a consequence of small phreatic explosions in the SW border of crater 3. This activity seems to be related to heat and fluid transfer from a shallow magmatic-hydrothermal reservoir with a minimal release of deep magmatic gases. This hydrothermal signature can be assumed as a "normal" gas composition during quiescent periods of Planchón-Peteroa volcano.

The phase 2 corresponds to the more intense eruptive phase along all eruptive period, with columns reaching up to 3,000 $\mathrm{m}$ of height, plumes dispersed at maximum distance of $344.8 \mathrm{~km}$ from the active crater and frequent tephra fall. Tephra fall deposits reached its greatest area at $10^{\text {th }}$ October $2010\left(1,265 \mathrm{~km}^{2}\right)$, and were exclusively constituted by no juvenile fragments. Some of these fragments were partially altered, being characterized by the presence of Fe-oxides, and less frequently $\mathrm{Cu}$-minerals. Considering the absence of juvenile products, there is no undoubtable evidences of new magmatic batch from depth that can be regarded.

The eruptive phases 3 and 4 were characterized by a permanent emission of gas/steam columns varying from 200 to $800 \mathrm{~m}$ of height that were occasionally associated with emission of ash columns of 1,0001,200 $\mathrm{m}$ height. Plumes were dispersed at variable distances (from 37 to $637.6 \mathrm{~km}$ ), with occasional fall of tephra. The componentry of the fall deposits was identical that the one during phase 2 , with sole presence of no juvenile fragments. The heat transfer probably began to progressively stabilise from phase 3 to 4 (which had increased previously from phase 1 to 3 , as observed by the thermodynamical review, with a temperature increasing from $\sim 290$ to $\sim 330^{\circ} \mathrm{C}$ ), producing less intense and less frequent explosive events. Fluids composition was still dominated by reduced hydrothermal fluids, but with a subtle increase of deep oxidized magmatic fluids, producing a limited increase in the concentration of typical magmatic species (e.g., $\mathrm{SO}_{2}$; Table 4). The re-establishment of the acid lake in the crater 3 is probably a consequence of repose period between phases 2 and 3, when rain and melting of glaciers during summer, in addition of water supply from from condensation of hydrothemarmal fluids, allowed the replenishment of this lake.

After the 2010-2011 eruptive period, the volcanic activity was characterized by a permanent low height steam column (50-100 m), but no explosive activity was observed. Fluid composition during March 2012 
shows a dramatic increase of the magmatic component contents (e.g., $\mathrm{SO}_{2}, \mathrm{HCl}$ and $\mathrm{HF}$ ) which is accompanied by higher oxidizing conditions $\left(\mathrm{R}_{\mathrm{H}}=-3.25\right)$ and separation of steam at higher pressure ( $\sim 60$ bar; Fig. 12a and b). In consequence, once the eruptive period finished, release of magmatic fluids from a deep reservoir to the surface become effective, but without eruptive activity associated.

A key observation for understanding the mechanisms responsible for the eruption pattern and the recorded geochemical evolution of the fumarolic fluids at Planchón-Peteroa volcano could be related to the N-S orientation of the subsidence that affected the neighboring volcanic systems (e.g., Calabozos caldera) within months of the $27^{\text {th }}$ February 2010 MW 8.8 Maule earthquake (Pritchard et al., 2013). Planchón-Peteroa volcano is emplaced along a NNE-SSW high-angle $\left(>60^{\circ}\right)$ thrust fault which is favorably orientated for dextral shear with respect to the prevailing stress field affecting the Andean region (i.e., subhorizontal maximum principal compressive stress- $\sigma_{\text {Hmax }}$ trending N $60^{\circ}$ E; Farías, 2007; Cembrano and Lara, 2009). Correspondingly, the N-S orientation of the subsidence reported by Pritchard $e t$ al. (2013) may reflect the long-term feature, i.e., the shape of both the hydrothermal-magmatic reservoir and the underlying magmatic system. As Pritchard et al. (2013) suggested, the existing NNE-striking magmatic and hydrothermal systems (e.g., Calabozos caldera) would have been disturbed by the static and dynamic stress change of the Maule earthquake through the formation and/or reactivation of numerous cracks suitably oriented with respect to the NNE-SSW co-seismic stress field, allowing fluids and heat to escape from the magmatic-hydrothermal reservoirs. According to this hypothesis, the enhanced fumarolic activity observed during the $1^{\text {st }}$ eruptive phase (February 2010) should be a consequence of this intense escape of fluids from a reservoir where vapour and liquid were in equilibrium at a $\mathrm{T} \sim 275^{\circ} \mathrm{C}$, a pressure $>60$ bar and redox conditions slightly more oxidized $\left(\mathrm{R}_{\mathrm{H}} \approx-3.1\right)$ that those dictated by the $\mathrm{FeO} /$ $\mathrm{FeO}_{1.5}$ buffer (Fig. 12a). Such fluid releases would have drastically reduced the fluid pressure within the magmatic-hydrothermal reservoir causing the phreatic eruptions observed during the $1^{\text {st }}$ eruptive phase, but without an evident surface deformation.

In March 2011, acid gases content at crater fumaroles, as well as the $\mathrm{CO}_{2} / \mathrm{CH}_{4}$ and $\mathrm{N}_{2} / \mathrm{Ar}$ ratios slightly increased compared to those observed in February 2010 (Table 4), whereas $R_{H}$ values remained relatively constant. These temporal changes suggest that after an almost uninterrupted release of fluids from the magmatic-hydrothermal reservoir during the 2010 unrest episode (Fig. 12a), a partial consumption of the liquid-dominated system overlying the magmatic zone would have occurred (e.g., Chiodini et al., 2004). Such process is clearly observed in figure $11 \mathrm{~b}$ where

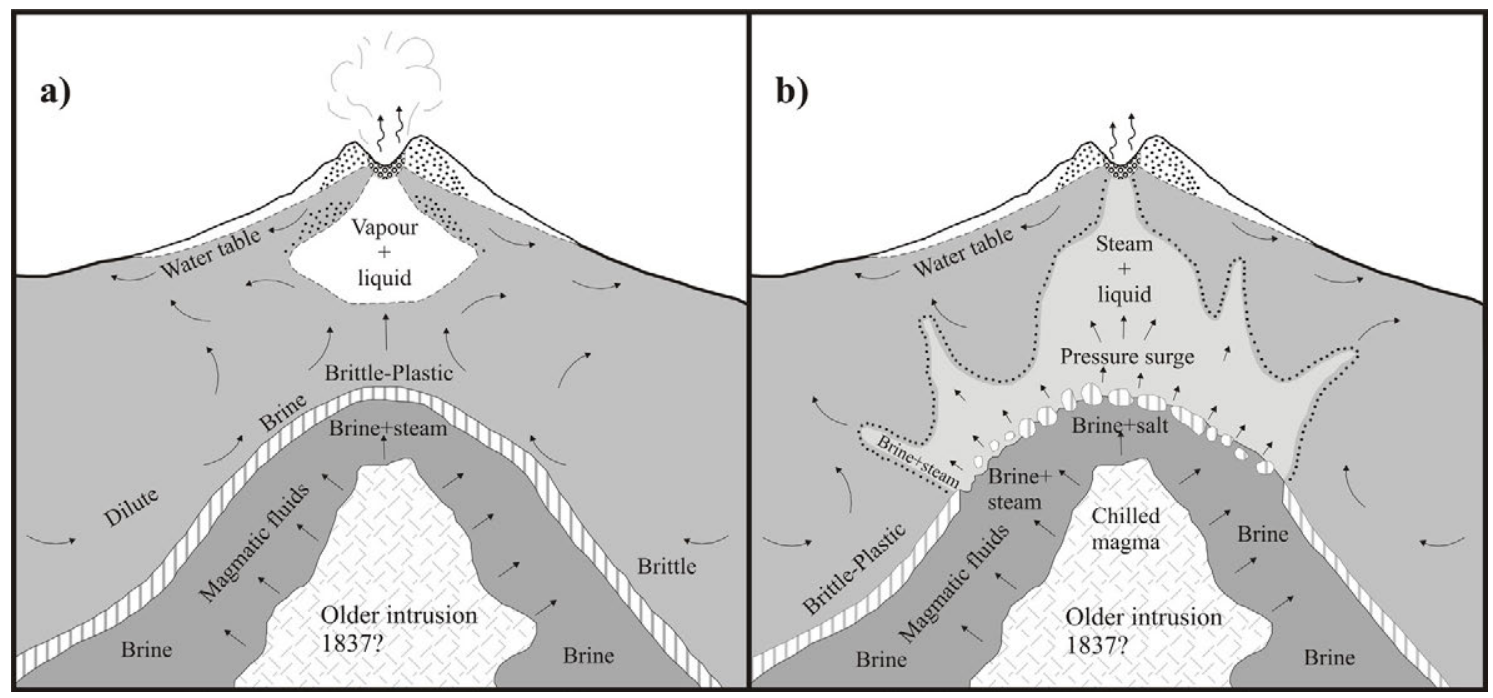

FIG. 12. Schematic models of the transition from a hydrothermal-magmatic episode (c) to magmatic episode (d) at Planchón-Peteroa volcano caused by a temporary breaching of a normally self-sealed zone (modified from Fournier, 2007). See text for discussion. 
fumarolic gases sampled in March 2011 plot close to the pure liquid equilibrium line at $\mathrm{T}_{\mathrm{o}} \sim 350^{\circ} \mathrm{C}$, being this composition typical of vapors separated from a liquid reservoir when complete vaporization of the liquid zone occurs (e.g., Guagua Pichincha-Ecuador, Montserrat-West Indies, Aguashuca-Salvador, Tambora-Indonesia; Chiodini and Marini, 1998).

Continuation of the degassing process from the magmatic-hydrothermal reservoir would have led to a total consumption of the liquid-dominated systems, lowering abruptly the load experienced by the underlying magmatic chamber at Planchón-Peteroa (e.g., Fournier, 2007). Fournier (2007) argued that such process may induce rapid vesiculation within the water-saturated magma, resulting in the rupture of the self-sealed zone surrounding the magmatic body (i.e., sealing carapace; Fisher et al., 1996), and consequently a rapid loss of the exsolved fluids from the crystallizing magma and that flashed from any brine that had previously resided in plastic rock beneath the self-sealed zone would have occurred (Fig. 12b). The increasing seismic activity observed from October 2011 to February 2012 at Planchón-Peteroa volcano and its surroundings (SERNAGEOMIN, $2011 \mathrm{c}^{3}$ and $\mathrm{d}^{4} ; 2012 \mathrm{a}^{5}, \mathrm{~b}^{6}$ and $\mathrm{c}^{7}$ ) along with the clear magmatic signature observed in March 2012 at the crater fumaroles is consistent with this hypothesis (e.g., Fournier, 2007). Discharge of magmatic fluid across a narrow ring directly into brittle rock where hydrostatic pressure conditions prevails (Fig. 12a) strongly favors the evolution of $\mathrm{SO}_{2}$-rich and $\mathrm{H}_{2} \mathrm{~S}$-poor fluids, explaining the relatively high $\mathrm{SO}_{2} / \mathrm{H}_{2} \mathrm{~S}$ ratios measured ( $>2$; Table 4$)$ at the crater fumaroles during March 2012 (e.g., Fournier, 2007; Liotta et al., 2010). The relatively low $\mathrm{HCl}$ contents $(<14.1 \mathrm{mmol} / \mathrm{mol})$ measured during the "magmatic" stage at the crater fumaroles may be a consequence of the relatively low temperature at which the vapor phase was separated $\left(\mathrm{T}_{\mathrm{S}}<275^{\circ} \mathrm{C}\right)$, as $\mathrm{HCl}_{(0)}$ is preferentially partitioned into the liquid phase at $\mathrm{T}<400{ }^{\circ} \mathrm{C}$ (Fournier, 2007). A similar scenario would have also occurred during the $2^{\text {nd }}$ eruptive phase, where a sudden rupture of the self-sealed zone would have caused the observed phreatic eruptions (Fig. 12b).

\section{Conclusions}

We conclude that the eruptive period 20102011 of Planchón-Peteroa volcano is related to the increasing of heat and mass transfer from shallow hydrothermal-deep magmatic sources, perhaps favoured by the formation and/or reactivation of cracks produced by the Maule earthquake $\left(27^{\text {th }}\right.$ February 2010). The chemistry of the crater fumaroles suggests two distinct phases of volcanic activity at PlanchónPeteroa: 1) During the 2010-2011 unrest episode, the crater fumaroles had a composition typically of volcanic emissions in magmatic-hydrothermal stages, dominated by $\mathrm{CO}_{2}\left(\mathrm{H}_{2} \mathrm{~S}\right)$ and with comparable amounts of hydrothermal- and magmatic- related components such as $\mathrm{CH}_{4}$, and $\mathrm{SO}_{2}, \mathrm{HCl}$ and $\mathrm{HF}$, respectively. The $\mathrm{CO}_{2} / \mathrm{CH}_{4}$ ratios slightly increased from 3,300-4,100, in February 2010, to 7,400-10,700, in March 2011, likely resulting from the evolution of the hydrothermal-magmatic reservoir to higher temperatures and/or oxidizing conditions. 2) In March 2012, the $\mathrm{SO}_{2}, \mathrm{HCl}$ and $\mathrm{HF}$ abundances of the crater fumaroles increased by three folds, indicating that magmatic fluids overwhelmed the previous "hydrothermal-magmatic signature". The change from a magmatic-hydrothermal stage to a subsequent phase dominated by magmatic components, seems to be a consequence of a degassing episode from a relatively "old" (i.e., highly degassed) magma body. The explosive activity dominated by phreatic eruptions, which ejected exclusively no juvenile material. Tephra fall deposit minimum volume was calculated in $0.0088 \mathrm{~km}^{3}$, and following Legros (2000) methodology, considering the $0.5 \mathrm{~cm}$ isopach and a density of $2,61 \mathrm{~g} / \mathrm{cm}^{3}$, the erupted mass calculated was $2.3 \times 10^{10} \mathrm{~kg}$. According to the classification proposed by Pyle (2000), the eruptive activity of Planchón-Peteroa volcano for 2010-2011 period, had a magnitude (M) 3.36, corresponding to a VEI 1-2 based in the classification of Newhall and Self (1982). The composition of tephra ash deposit and its physical parameters (e.g., area, volume, grain size distribution) are consistent with the low magnitude of the eruption, low VEI value and eruption type (phreatic). The low magnitude of these phreatic explosions prevented the glacier melting, avoiding the lahars formation, which could affect the Teno and Claro rivers, like occurred during previous events (González-Ferrán, 1995; Naranjo et al., 1999).

\section{Acknowledgments}

This work was funded by "Proyecto de Iniciación FONDECYT No. 11100372: Role of climate, continental crust and subducted sediments in the degassing style and fluid composition of Andean volcanoes" (F. Aguilera). 
V. Marfull (Centro de Investigación y Difusión de Volcanes de Chile, Proyecto Archivo Nacional de Volcanes), R. Tormey (Entrix Inc.), Gendarmería Argentina (Paso Vergara) and local witnesses gave us important field-based information that improve our database related with this eruptive process. Finally we would like to acknowledge to C. González, M. Inostroza and S. Layana for their help during satellite images processing and tephra samples analysis. Our acknowledgements to Andean Geology editor (Dr. L. Lara) by its valuable comments of the manuscript.

\section{References}

Aguilera, F.; Benavente, O.; Gutiérrez, F.; Agusto, M.; Caselli, A. 2011. Distribution of gas-water discharges and current geochemical survey along PlanchónPeteroa-Azufre Volcanic Complex, southern Chile. In Gas Workshop, No. 11, 1: p. 5. Kamchatka.

Agusto, M.; Tassi, F.; Caselli, A.; Vaselli, O.; Rouwet, D.; Capaccioni, B.; Caliro, S.; Chodini, G.; Darrah, T. 2013. Gas geochemistry of the magmatic-hydrothermal fluid reservoir in the Copahue-Caviahue Volcanic Complex (Argentina). Journal of Volcanology and Geothermal Research 257: 44-56.

Ayele, A.; Jaques, E.; Kassim, M.; Kidane, T.; Omar, A.; Tait, S.; Nercessian, A.; de Chabalier, J-B.; King, G. 2007. The volcano-seismic crisis in Afar, Ethiopia, starting September 2005. Earth and Planetary Science Letters 255: 177-187.

Benavente, O. 2010. Actividad hidrotermal asociada a los complejos volcánicos Planchón-Peteroa y Descabezado Grande-Quizapu-Cerro Azul, 36 y $37^{\circ} \mathrm{S}$, Zona Volcánica Sur, Chile. Ph.D. Thesis (Unpublished), Universidad de Chile, Departamento de Geología: 203 p.

Benavente, O. 2015. Origen y naturaleza de los fluidos en los sistemas volcánicos e hidrotermales activos de los Andes de Chile Central (32.5-36 ${ }^{\circ}$ S). Ph.D. Thesis (Unpublished), Universidad de Chile, Departamento de Geología: 218 p.

Benavente, O.; Tassi, F.; Aguilera, F.; Agusto, M.; Caselli, A.; Gutiérrez, F.; Vaselli, O.; Reich, M. 2013. Central Chile volcanic lakes: The Tupungatito and Peteroa acid crater lakes. In Crater Volcanic Lakes Workshop 1: p. 25.

BGVN. 1991. Ash eruption to $1 \mathrm{~km}$; drinking water contaminated; fish killed. Smithsonian Institute, Bulletin of the Global Volcanism Network 16 (01): 1 p.

BGVN. 1999. Unrest including ashfall and $\mathrm{SO}_{2}$ emissions in November 1998. Smithsonian Institute, Bulletin of Global Volcanism Network 24 (03): 1 p.
BGVN. 2010. Small eruptions begin in September 2010 after 12 years of quiet. Smithsonian Institute, Bulletin of Global Volcanism Network 35 (11): 1 p.

Bryan, C.; Sherburn, S. 1999. Seismicity associated with the 1995-1996 eruptions of Ruapehu volcano, New Zealand: narrative and insights into physical processes. Journal of Volcanology and Geothermal Research 90: 1-18.

Cembrano, J.; Lara, L. 2009. The link between volcanism and tectonics in the southern volcanic zone of the Chilean Andes: A review. Tectonophysics 471: 96-113.

Chiodini, G.; Marini, L. 1998. Hydrothermal gas equilibria: The $\mathrm{H}_{2} \mathrm{O}-\mathrm{H}_{2}-\mathrm{CO}_{2}-\mathrm{CO}-\mathrm{CH}_{4}$ system. Geochimica et Cosmochimica Acta 62: 2673-2687.

Chiodini, G.; Avino, R.; Brombach, T.; Caliro, S.; Cardellini, C.; De Vita, S.; Frondini, F.; Granirei, D.; Marotta, E.; Ventura, G. 2004. Fumarolic and diffuse soil degassing west of Mount Epomeo, Ischia, Italy. Journal of Volcanology and Geothermal Research 133: 291-309.

Chouet, B.; Page, R.; Stephens, C.; Lahr, J.; Power, J. 1994. Precursory swarms of long-period events at Redoubt volcano (1989-1990), Alaska: Their origin and use as a forecasting tool. Journal of Volcanology and Geothermal Research 62: 95-135.

Christenson, B. 2000. Geochemistry of fluids associated with the 1995-1996 eruption of Mt. Ruapehu, New Zealand: signatures and processes in the magmatichydrothermal system. Journal of Volcanology and Geothermal Research 97: 1-30.

Davidson, J. 1974. A Quaternary volcanic mudflow (lahar) down the Claro and Teno valleys from Planchón volcano. In International Symposium of Volcanology, Volume 1: 14-15. Santiago.

Farías, M. 2007. Tectónica de la erosión en la evolución del relieve de los Andes de Chile Central durante el Neógeno. Ph.D. Thesis (Unpublished), Universidad de Chile, Departamento de Geología: 194 p.

Fischer, T.; Arehart, G.B.; Sturchio, N.C; Williams, S.N. 1996. The relationship between fumarole gas composition and eruptive activity at Galeras volcano, Colombia. Geology 24: 531-534.

Fischer, T.P.; Sturchio, N.C.; Stix, J.; Arehart, G.B.; Counce, D.; Williams, S.N. 1997. The chemical and isotopic composition of fumarolic gases and spring discharges from Galeras Volcano, Colombia. Journal of Volcanology and Geothermal Research 77: 229-253.

Fournier, R. 2007. Hydrothermal systems and volcano geochemistry. In Volcano Deformation-Geodetic moni- 
toring Techniques (Dzurisin, D.; editor). Springer-Praxis Books in Geophysical Sciences: 323-341. Berlin.

Giggenbach, W.F. 1987. Redox processes governing the chemistry of fumarolic gas discharges from White island, New Zealand. Applied Geochemistry 2: 143-161.

Giggenbach, W.F. 1991. Chemical techniques in geothermal exploration. In Application of geochemistry in geothermal reservoir development (D'Amore, F.; editor). UNITAR/UNDP Center on Small Energy Resources (United Nations Institute for Training and Research/United Nations Development Programme): 119-144. Rome.

Giggenbach, W.F. 1992. Isotopic shifts in waters from geothermal and volcanic systems along convergent plate boundaries and their origin. Earth and Planetary Sciences Letters 113: 495-510.

Giggenbach, W.F. 1993 Redox control of gas compositions in Philippine volcanic-hydrothermal systems. Geothermics 22: 575-587.

Giggenbach, W.F. 1996. Chemical composition of volcanic gases. In Monitoring and mitigation of volcano hazards (Scarpa, R.; Tilling, R.; editors). Springer-Verlag: 222-256. Berlin.

Giggenbach, W.F.; Goguel, R.L. 1989. Collection and analysis of geothermal and volcanic water and gas discharges. Report (Unpublished). Chemistry Division, Department of Scientific and Industrial Research (DSIR)-Petone: 1-81. New Zealand.

González-Ferrán, O. 1995. Volcanes de Chile. Instituto Geográfico Militar: 639 p. Santiago.

Gutiérrez, F.; Lemus, M.; Parada, M.; Benavente, O.; Aguilera, F. 2012. Contribution of ground altitude difference to thermal anomaly detection using satellite images: Application to volcanic/geothermal complexes in the Andes of Central Chile. Journal of Volcanology and Geothermal Research 237-238: 69-80.

Haller, M.; Risso, C. 2011. La erupción del volcán Peteroa (35 $15^{\circ}$ 'S, $\left.70^{\circ} 18^{\prime} \mathrm{O}\right)$ del 4 de Septiembre de 2010. Revista de la Asociación Geológica Argentina 68: 295-305.

Haller, M.; Nullo, F.; Proserpio, C.; Parica, P.; Cagnoni, M.; Walker, J. 1985. Major element geochemistry of early Tertiary Andean volcanics $\left(34^{\circ}-36^{\circ} \mathrm{S}\right)$. Comunicaciones 35: 97-100.

Haller, M.; Mendía, J.; Ostera, H. 1991. Mapa preliminar de riesgo en la vertiente Argentina del volcán Peteroa. In Congreso Geológico Chileno, No. 6, Actas: 355 358. Viña del Mar.

Haller, M.; Ostera, H.; Pesce, A.; Gardini, M.; Folguera, A. 1994. Vulcanoestratigrafía reciente y eruptividad del
Volcán Peteroa. In Congreso Geológico Chileno, No. 7, Actas: 319-323. Concepción.

Harris, A.J.L.; Flynn, L.P.; Dean, K.; Pilger, E.; Wooster, M.; Okubo, C.; Mouginis-Mark, P.; Garbeil, H.; De la Cruz Reyna, S.; Thornber, C.; Rothery, D.; Wright, R. 2000. Real-time Monitoring of Volcanic Hot Spots with Satellites, Remote Sensing of Active Volcanism. American Geophysical Union Monograph Series 116: 139-159.

Legros, F. 2000. Minimum volume of a tephra fallout deposit estimated from a single isopach. Journal of Volcanology and Geothermal Research 96: 25-32.

Liotta, M.; Paonita, A.; Caracausi, A.; Martelli, M.; Rizzo, A.; Favara, R. 2010. Hydrothermal processes governing the geochemistry of the crater fumaroles at Mount Etna volcano (Italy). Chemical Geology 278: 92-104.

López-Escobar, L.; Cembrano, J.; Moreno, H. 1995. Geochemistry and tectonics of the Chilean Southern Andes basaltic Quaternary volcanism (37-46 $\left.{ }^{\circ} \mathrm{S}\right)$. Revista Geológica de Chile 22 (2): 219-234. doi: 10.5027/andgeoV22n2-a06.

MacPhail, D. 1973. The geomorphology of the rio Teno lahar, central Chile. Geographical Review 63: 517-532.

Montegrossi, G.; Tassi, F.; Vaselli, O.; Buccianti, A.; Garofalo, K. 2001. Sulfur species in volcanic gases. Analytical Chemistry 73: 3709-3715.

Murcia, H.; Cortés, G.; Mendoza, B. 2008. Métodos e integración de análisis granulométrico para depósitos volcanoclásticos. Instituto Colombiano de Geología y Minería (INGEOMINAS) 42: 129-139.

Naranjo, J. 2012. Principales etapas evolutivas holocenas del volcán Planchón y su reactivación relacionada al megasismo del 27 de Febrero de 2010. In Congreso Geológico Chileno, No. 13, Actas: 440-441. Antofagasta.

Naranjo, J.; Haller, M.; Ostera, H.; Pesce, A.; Sruoga, P. 1999. Geología y Peligros del Complejo Volcánico Planchón-Peteroa, Andes del Sur (3515'S), Región del Maule, Chile-Provincia de Mendoza, Argentina. Servicio Nacional de Geología y Minería, Boletín 52: $55 \mathrm{p}$.

Naranjo, J.; Haller, M. 1997. Actividad explosiva postglacial del complejo volcánico Planchón-Peteroa, $35^{\circ} 15^{\prime}$ S. In Congreso Geológico Chileno, No. 6, Actas: 357-360. Antofagasta.

Naranjo, J.; Haller, M. 2002. Erupciones principalmente explosivas del volcán Planchón, Andes del sur (35 ${ }^{\circ} 15^{\prime}$ S). Revista Geológica de Chile 29 (1): 93-111. doi: 10.5027/andgeoV29n1-a06. 
Newhall, C.G.; Self, S. 1982. The Volcanic Explosivity Index (VEI): an estimate of explosive magnitude for historical volcanism. Journal of Geophysical Research 87: 1231-1238.

Pasternack, G.B.; Varekamp, J.C. 1994. The geochemistry of the Keli Mutu crater lakes, Flores, Indonesia. Geochemical Journal 28: 243-262.

Pritchard, M.E.; Jay, J.A.; Aron, F.; Henderson, S.T.; Lara, L.E. 2013. Subsidence at southern Andes volcanoes induced by the 2010 Maule, Chile earthquake. Nature Geoscience 6: 632-636.

Pyle, D.M. 2000. Sizes of volcanic eruptions. In Encyclopedia of Volcanoes (Sigurdsson, H.; Houghton, B.F.; McNutt, S.R.; Rymer, H.; Stix, J.; editors). Academic Press: 263-270. London.

Reagan, M.; Gill, J.; Malavassi, E.; García, M. 1987. Changes in magma composition at Arenal volcano, Costa Rica, 1968-1985: Real-time monitoring of open-system differentation. Bulletin of Volcanology 49: 415-434.

Saltori, O. 2014. Geoquímica de fluidos del Complejo Volcánico Planchón-Peteroa-Azufre, Zona Volcánica Sur, Chile. Memoria de Título (Unpublished), Universidad de Atacama, Departamento de Geología: 148 p.

Sellés, D.; Rodríguez, C.; Dungan, M.; Naranjo, J.; Gardeweg, M. 2004. Geochemistry of Nevado de Longavi volcano $\left(36.2^{\circ} \mathrm{S}\right)$ : A compositionally atypical arc volcano in the Southern Volcanic Zone of the Andes. Revista Geológica de Chile 31 (2): 293-315. doi: 10.5027/andgeoV31n2-a08.

Sepúlveda, F.; Lahsen, A.; Powell, T. 2007. Gas geochemistry of the Cordón Caulle geothermal system, southern Chile. Geothermics 36: 389-420.

Taran, Y.; Pokrovsky, B.; Esikov, A. 1989. Deuterium and oxigen-18 in fumarolic steam and amphiboles from some Kamchatka volcanoes: "andesitic waters". Doklady Akademii Nauk SSSR 304: 440-443.

Tassi, F.; Aguilera, F.; Benavente, O.; Paonita, A.; Chiodini, G.; Caliro, S.; Agusto, M.; Gutiérrez, F.; Capaccioni, B.; Vaselli, O.; Caselli, A.; Saltori, O. In press. Geochemistry of fumarolic fluids from Peteroa volcano (Argentina-Chile) in 2010-2015: Insights into compositional changes related to the fluid source region(s). Chemical Geology.

Tassi, F.; Vaselli, O.; Papazachos, C.B.; Giannini, L.; Chiodini, G.; Vougioukalakis, G.E.; Karagianni, E.; Vamvakaris, D.; Panagiotopoulos, D. 2013. Geochemical and isotopic changes in the fumarolic and submerged gas discharges during the 2011-2012 unrest at Santorini caldera (Greece). Bulletin of Volcanology 75: 1-15.

Tassi, F.; Capaccioni, B.; Vaselli, O. 2014. Compositional spatial zonation and 2005-2013 temporal evolution of the hydrothermal-magmatic fluids from the submarine fumarolic field at Panarea Island (Aeolian Archipielago, southern Italy). Journal of Volcanology and Geothermal Research 277: 41-50.

Tormey, D. 1989. Geology and Geochemistry of the active Azufre-Planchon-Peteroa volcanic center $35^{\circ} 15^{\prime} \mathrm{S}$, Southern Andes: Implications for Cordilleran arc magmatism. Ph.D. Thesis (Unpublished), Massachusetts Institute of Technology: $331 \mathrm{p}$.

Tormey, D. 2010. Managing the effects of accelerated glacial melting on volcanic collapse and debris flows: Planchón-Peteroa volcano, southern Andes. Global and Planetary Change 74: 82-90.

Tormey, D.; Frey, F.; López, L. 1995. Geochemistry of the Active Azufre-Planchón-Peteroa Volcanic Complex, Chile (35 15 'S): Evidence for Multiple Sources and Processes in a Cordilleran Arc Magmatic System. Journal of Petrology 36: 265-298.

Trombotto Liaudat, D.; Penas, P.; Aloy, G. 2014. Impact of volcanic processes on the cryospheric system of the Peteroa Volcano, Andes of southern Mendoza, Argentina. Geomorphology 208: 74-87.

Varekamp, J.; Ouimette, A. 2001. Crater lake brines as modern analogs of ore-transporting fluids. In Geological Society of America (GSA), Annual Meeting 33 (6): p. 359. Boston.

Varekamp, J.C.; Ouimette, A.; Kreulen, R. 2004. The magmato-hydrothermal system of Copahue volcano, Argentina. In Proceedings International Conference on Water-Rock Interaction, No. 11, Balkema Publishers 1: 215-218. Leiden.

Varekamp, J.; Ouimette, A.; Herman, S.; Bermúdez, A.; Delpino, D. 2001. Hydrothermal element fluxes from Copahue, Argentina: A "beehive" volcano in turmoil. Geology 29: 1059-1062.

Vaselli, O.; Tassi, F.; Montegrossi, G.; Capaccioni, B.; Giannini, L. 2006. Sampling and analysis of fumarolic gases. Acta Vulcanologica 18: 65-76.

Vaselli, O.; Tassi, F.; Duarte, E.; Fernández, E.; Poreda, R.J.; Delgado-Huertas, A. 2010. Evolution of fluid geochemistry at the Turrialba volcano (Costa Rica) from 1998 to 2008. Bulletin of Volcanology 72: 397-410.

Vélez, L.; Euillades, P.; Caselli, A.; Blanco, M.; Martínez, J. 2011. Deformation of Copahue volcano: Inversion of InSAR data using a genetic algorithm. 
Journal of Volcanology and Geothermal Research 202: $117-126$.

Villemant, B.; Komorowski, J.; Dessert, C.; Michel, A.; Crispi, O.; Hammouya, G.; Beauducel, F.; de Chebalier, J-B. 2014. Evidence for a new shallow magma instrusion at La Soufriere of Guadaloupe (Lesser Antilles). Insights from long-term geochemical monitoring of halogen-rich hydrothermal fluids. Journal of Volcanology and Geothermal Research 285: $247-277$.
Walker, G.P.L. 1971. Grain-size characteristics of pyroclastic deposits. Journal of Geology 79: 696-714.

Walter, T.R.; Wang, R.; Acocella, V.; Neri, M.; Grosser, H.; Zschau, J. 2009. Simultaneous magma and gas eruptions at three volcanoes in southern Italy: an earthquake trigger? Geology 37: 251-254.

Wohletz, K.H. 1983. Mechanisms of hydrovolcanic pyroclastic formation: grain-size, scanning electron microscopy, and experimental studies. Journal of Volcanology and Geothermal Research 17: 31-63.

Manuscript received: August 25, 2014; revised/accepted: September 21, 2015; available online: September 23, 2015. 